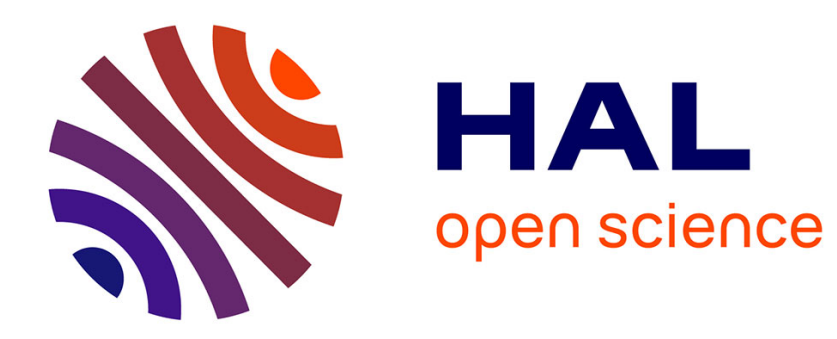

\title{
Interpretable Groups in Mann Pairs
}

Haydar Göral

\section{- To cite this version:}

Haydar Göral. Interpretable Groups in Mann Pairs. Archive for Mathematical Logic, 2017. hal01197019v3

\section{HAL Id: hal-01197019 \\ https://hal.science/hal-01197019v3}

Submitted on 23 Oct 2017

HAL is a multi-disciplinary open access archive for the deposit and dissemination of scientific research documents, whether they are published or not. The documents may come from teaching and research institutions in France or abroad, or from public or private research centers.
L'archive ouverte pluridisciplinaire HAL, est destinée au dépôt et à la diffusion de documents scientifiques de niveau recherche, publiés ou non, émanant des établissements d'enseignement et de recherche français ou étrangers, des laboratoires publics ou privés. 


\title{
INTERPRETABLE GROUPS IN MANN PAIRS
}

\author{
HAYDAR GÖRAL
}

\begin{abstract}
In this paper, we study an algebraically closed field $\Omega$ expanded by two unary predicates denoting an algebraically closed proper subfield $k$ and a multiplicative subgroup $\Gamma$. This will be a proper expansion of algebraically closed field with a group satisfying the Mann property, and also pairs of algebraically closed fields. We first characterize the independence in the triple $(\Omega, k, \Gamma)$. This enables us to characterize the interpretable groups when $\Gamma$ is divisible. Every interpretable group $H$ in $(\Omega, k, \Gamma)$ is, up to isogeny, an extension of a direct sum of $k$ rational points of an algebraic group defined over $k$ and an interpretable abelian group in $\Gamma$ by an interpretable group $N$, which is the quotient of an algebraic group by a subgroup $N_{1}$, which in turn is isogenous to a cartesian product of $k$-rational points of an algebraic group defined over $k$ and an interpretable abelian group in $\Gamma$.
\end{abstract}

\section{INTRODUCTION}

Let $\Omega$ be an algebraically closed ambient field, the field $k$ be a proper subfield of $\Omega$ and $\Gamma$ be a multiplicative subgroup of $\Omega^{\times}$. We begin by defining a uniform version of the Mann property which was introduced in [5]. First, we recall the Mann property. Consider an equation

$$
a_{1} x_{1}+\cdots+a_{n} x_{n}=1
$$

with $n \geq 1$ and $a_{i} \in k^{\times}$. A solution $\left(g_{1}, \ldots, g_{n}\right)$ of this equation is called non-degenerate if for every non-empty subset $I$ of $\{1,2, \ldots, n\}$, the sum $\sum_{i \in I} a_{i} g_{i}$ is not zero. We say that $\Gamma$ has the Mann property over $k$ if every such equation (1) has only finitely many non-degenerate solutions in $\Gamma$. It was proved by L. van den Dries and A. Günaydın [4, Proposition 5.6] that the Mann property is global, which means if we choose $a_{i}$ to be in $\Omega$ in (1) then this still gives finitely many non-degenerate solutions in $\Gamma$. We say that $(k, \Gamma)$ is a Mann pair if for all $n$ there is a finite subset $\Gamma(n)$ of $\Gamma$ such that for all $a_{1}, \ldots, a_{n}$ in $k^{\times}$all non-degenerate solutions of (1) in $\Gamma$ lie in $\Gamma(n)$. This is a uniform version of the Mann property and the finiteness condition does not depend on the given parameters $a_{1}, \ldots, a_{n}$. In particular, the group $\Gamma$ has the Mann property if $(k, \Gamma)$ is a Mann pair. Given $a_{1}, \ldots, a_{n}$ in $k^{\times}$, let $N d\left(a_{1}, \ldots, a_{n}\right)$ be the set of all non-degenerate solutions of the equation (1) lying in $\Gamma$. Therefore, the group $\Gamma$ has the Mann property if and only if for each tuple $\left(a_{1}, \ldots, a_{n}\right)$ the set $N d\left(a_{1}, \ldots, a_{n}\right)$ is finite, and $(k, \Gamma)$ is a Mann pair if and only if for each $n \geq 1$ the whole union

$$
\bigcup_{\left(a_{1}, \ldots, a_{n}\right) \in k^{\times}} N d\left(a_{1}, \ldots, a_{n}\right)
$$

is finite.

1991 Mathematics Subject Classification. 03C45.

Key words and phrases. model theory, stability, mann pairs.

Partially supported by ValCoMo (ANR-13-BS01-0006) and MALOA (PITN-GA-2009-238381). 
Observe that if $(k, \Gamma)$ is a Mann pair then taking $n=1$ in the definition we see that the intersection $k \cap \Gamma$ is finite, thus the intersection is a finite subset of the group of roots of unity in $\Omega$. Therefore, if $(k, \Gamma)$ is a Mann pair and if $\Gamma$ is infinite, then most of the elements in $\Gamma$ are transcendental over the field $k$. In [15], H. Mann showed that the set of complex roots of unity $\mu$ has the Mann property and moreover the pair $(\mathbb{Q}, \mu)$ is a Mann pair. His proof is effective. Later on, H. Mann's result was generalized in the 1980's and it was proved that any multiplicative group of finite rank ( $\mu$ has rank 0) in any field of characteristic zero has the Mann property; see [7]. To illustrate, every finitely generated multiplicative subgroup of $\mathbb{C}^{\times}$has the Mann property, such as $2^{\mathbb{Z}} 3^{\mathbb{Z}}$. The pair $(\overline{\mathbb{Q}}, \exp (\overline{\mathbb{Q}}))$ is a Mann pair by Lindemann's theorem. Note that the group $\exp (\overline{\mathbb{Q}})$ is of infinite rank. In [5, Theorem 1.1], L. van den Dries and A. Günaydın proved that when $k$ is algebraically closed, if the intersection $k \cap \Gamma$ is trivial and if $\Gamma$ is of finite rank then $(k, \Gamma)$ is a Mann pair. This provides substantial examples of Mann pairs, such as $\left(\mathbb{C}, t^{\mathbb{Z}}\right)$ where $t$ is an indeterminate.

Now fix an algebraically closed field $\Omega_{0}$. Moreover, we also fix an algebraically closed proper subfield $k_{0}$ of $\Omega_{0}$ and an infinite multiplicative subgroup $\Gamma_{0}$ of $\Omega_{0}$ where $\left(k_{0}, \Gamma_{0}\right)$ is a Mann pair. By the triple $\left(\Omega_{0}, k_{0}, \Gamma_{0}\right)$ we actually mean the structure $\left(\Omega_{0}, k_{0}, \Gamma_{0},+,-, \cdot, 0,1\right)$. Thus, our language is $\left\{+,-, \cdot, 0,1, P_{1}, P_{2}\right\}$ where $P_{1}$ and $P_{2}$ are unary predicates whose interpretations in $\Omega_{0}$ are $k_{0}$ and $\Gamma_{0}$ respectively. The model theory of the triple $\left(\Omega_{0}, k_{0}, \Gamma_{0}\right)$ was studied in [5,6] by L. van den Dries and A. Günaydın, where they proved that the theory $T h\left(\Omega_{0}, k_{0}, \Gamma_{0}\right)$ is stable and it is $\omega$-stable if $\Gamma_{0}$ is divisible [5, Proposition 8.3]. To study definable groups in the triple, we need to add the constants $k_{0} \cup \Gamma_{0}$ as they did in [5] to have certain subsets of $k^{m} \times \Gamma^{n}$ definable with parameters from $k_{0}$ and $\Gamma_{0}$; see Theorem (2.1). So our language $L_{t}$ throughout this article is $\left\{+,-, \cdot, 0,1, P_{1}, P_{2}\right\}$ together with the constants for each element of $k_{0} \cup \Gamma_{0}$. Let $T_{t}$ be the complete theory of $\left(\Omega_{0}, k_{0}, \Gamma_{0}\right)$ in the language $L_{t}$. Therefore, if $(\Omega, k, \Gamma)$ is a model of $T_{t}$, then $k$ and $\Gamma$ are elementary extensions of $k_{0}$ and $\Gamma_{0}$ respectively. Note also that, if $\Omega$ is an algebraically closed field containing $\Omega_{0}$ then $\left(\Omega, k_{0}, \Gamma_{0}\right)$ is an elementary extension of $\left(\Omega_{0}, k_{0}, \Gamma_{0}\right)$ by $[6,4.4]$ as they have the same $k$ and $\Gamma$ parts. Thus, L. van den Dries and A. Günaydin proved that $k \cup \Gamma$ is small in $\Omega$ [5, Lemma 8.2], which means that by changing the model (the triple) we may assume that $\Omega$ is $|k \cup \Gamma|^{+}$-saturated as a field. We also assume the triple is $\kappa$-saturated and strongly homogeneous for some uncountable cardinal $\kappa$. Throughout the paper, we will be working in this sufficiently saturated model $(\Omega, k, \Gamma)$ where the base field $\Omega$ is also $|k \cup \Gamma|^{+}$-saturated as a field.

The model theory of the pair $(\Omega, \Gamma)$ was analyzed by the author in $[9,10]$ in terms of stability. The model theory and definable groups in $(\Omega, k)$ were studied in $[20,1]$ and [2]. In this paper, our concern will be the triple $(\Omega, k, \Gamma)$ in the stability framework, and this corresponds to the third chapter of the author's $\mathrm{PhD}$ thesis [9]. We also investigate the connection between the triple $(\Omega, k, \Gamma)$ and the pairs $(\Omega, k)$ and $(\Omega, \Gamma)$. The triple $(\Omega, k, \Gamma)$ is a proper expansion of $(\Omega, k)$ (a pair of algebraically closed fields) and also $(\Omega, \Gamma)$ (an algebraically closed field with a distinguished group having the Mann property). We first focus on algebraic closure and forking in the triple. This allows us to characterize definable groups in the triple in terms 
of definable and interpretable groups in $\Omega, k$ and $\Gamma$. Moreover, we characterize interpretable groups following the approach of [2] and applying the group configuration theorem [11] from geometric stability theory. Note that the group configuration theorem also holds for $*$-definable groups [13]. More precisely, we prove the following result (see Theorem (5.13)):

Theorem A. (Definable Groups) Let $\Omega$ be an algebraically closed field, the field $k$ be a proper subfield of $\Omega$ which is also algebraically closed and $\Gamma$ be a multiplicative subgroup of $\Omega^{\times}$such that $(k, \Gamma)$ is a Mann pair. Any type-definable group in $(\Omega, k, \Gamma)$ is isogenous to a subgroup of an algebraic group. Moreover, any type-definable group $H$ is, up to isogeny, an extension by an algebraic group $V$ of the direct sum of the $k$-rational points of an algebraic group $V_{1}$ over $k$ and a type-interpretable abelian group $H_{1}$ in $\Gamma$ :

$$
0 \longrightarrow V(\Omega) \longrightarrow H \longrightarrow V_{1}(k) \times H_{1}(\Gamma) \longrightarrow 0
$$

When $\Gamma$ is divisible, the characterization of definable groups in the triple enables us to characterize interpretable groups. In particular, we conclude (see Theorem (6.12)):

Theorem B. (Interpretable Groups) Let $\Omega$ be an algebraically closed field, the field $k$ be a proper subfield of $\Omega$ which is also algebraically closed and $\Gamma$ be a divisible multiplicative subgroup of $\Omega^{\times}$ such that $(k, \Gamma)$ is a Mann pair. Every interpretable group $H$ in $(\Omega, k, \Gamma)$ is, up to isogeny, an extension of a direct sum of $k$-rational points of an algebraic group $V_{1}$ over $k$ and an interpretable abelian group $H_{1}$ in $\Gamma$ by an interpretable group $N$, which is a quotient of an algebraic group $W$ by a subgroup $N_{1}$ which in turn is isogenous to a cartesian product of $k$-rational points of an algebraic group $V_{2}$ over $k$ and an interpretable abelian group $\mathrm{H}_{2}$ in $\Gamma$ :

$$
0 \longrightarrow N \longrightarrow H \longrightarrow V_{1}(k) \times H_{1}(\Gamma) \longrightarrow 0
$$

with

$$
0 \longrightarrow V_{2}(k) \times H_{2}(\Gamma) \longrightarrow W \longrightarrow N \longrightarrow 0
$$

\section{Preliminaries and Notations}

Now we introduce more notations. For a substructure $A$ in the sense of the triple, we denote $k_{A}=A \cap k$ (the $k$-part of $A$ ) and $\Gamma_{A}=A \cap \Gamma$ (the $\Gamma$-part of $A$ ). $\operatorname{By} \operatorname{acl}(A)$, we mean the algebraic closure of $A$ in the field sense and $\operatorname{acl}_{t}(A)$ indicates the algebraic closure of $A$ in the triple $(\Omega, k, \Gamma)$. By $\downarrow$ we mean the algebraic independence in the pure field $\Omega$ and $\stackrel{t}{\downarrow}$ signifies the independence in the triple $(\Omega, k, \Gamma)$. Similarly, $\stackrel{P}{1}^{\perp}$ denotes the independence in the pair

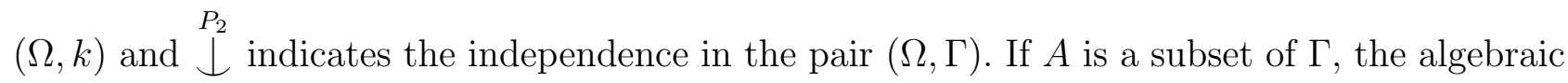
closure of $A$ in $\Gamma$ will be represented by $\operatorname{acl}_{\Gamma}(A)$. Let $a$ be a tuple in $\Omega$ and $B$ be a set of parameters. Unless otherwise stated, the type $\operatorname{tp}(a / B)$ denotes the type of $a$ over $B$ in the pure field sense. By $\operatorname{tp}^{t}(a / B)$ we mean the type of $a$ over $B$ in the sense of the triple. We use similar notations for $\operatorname{tp}_{k}$ and $\operatorname{tp}_{\Gamma}$ to indicate the types in $k$ and $\Gamma$ respectively. 
Let $E, F$ and $L$ be three fields where $L \subseteq E \cap F$. We say that $E$ is linearly disjoint from $F$ over $L$, denoted by $E \underset{L}{\stackrel{l d}{\downarrow}} F$, if each tuple $\left(a_{1}, \ldots, a_{m}\right)$ in $E^{m}$ which is linearly independent over $L$ is also linearly independent over $F$. The field extension $E \mid L$ is called regular if $E \underset{L}{\downarrow} \downarrow_{d}^{l d} \operatorname{acl}(L)$. Now we list some basic properties of linear disjointness which we will use frequently in the rest of the paper. For the details, we refer the reader to [8, Chapter 2] and [14, Chapter 8].

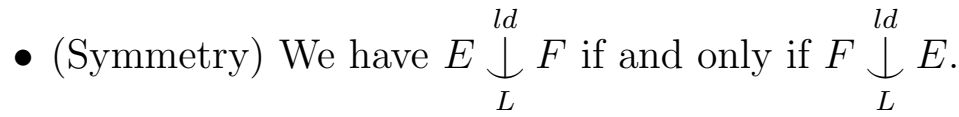

- (Transitivity) For a subfield $S$ of $F$ containing $L$, we have $E \underset{L}{\stackrel{l d}{\downarrow}} F$ if and only if $E S \underset{S}{\stackrel{l d}{\downarrow}} F$

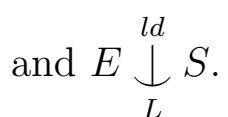

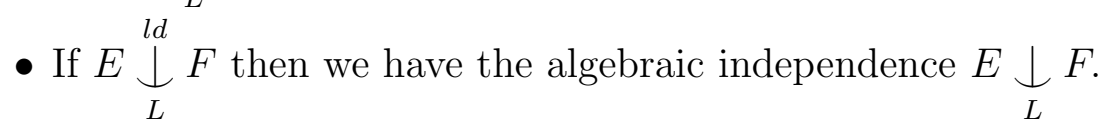

- If $L$ is an algebraically closed field, then the independence $E \underset{L}{\downarrow} F$ also implies $E \underset{L}{\stackrel{l d}{\downarrow}} F$.

Adding the constants for each element of $k_{0}$ and $\Gamma_{0}$ will be significant to control the parameters for definability, since we need algebraically closed structures to contain enough elements. The following theorem is in [5] and it states that $k$ and $\Gamma$ are orthogonal in the model-theoretic sense.

Theorem 2.1. [5, Theorem 1.2 and Remark in 8.3] For all $m, n \geq 1$, every definable subset of $k^{m} \times \Gamma^{n}$ definable in $(\Omega, k, \Gamma)$ is a finite union of sets $X \times Y$ with $X \subseteq k^{m}$ definable in the field $k$ and $Y \subseteq \Gamma^{n}$ definable in the group $\Gamma$. In other words, the induced structure on $(k, \Gamma)$ is itself. In particular, the set

$$
\Sigma_{n}=\left\{\left(k_{1}, \ldots, k_{n}, g_{1}, \ldots, g_{n}\right): k_{1} g_{1}+\cdots+k_{n} g_{n}=0\right\} \subseteq k^{n} \times \Gamma^{n}
$$

is a finite union of sets $X \times Y$ with $X \subseteq k^{n}$ definable in the field $k$ with parameters from $k_{0}$ and $Y \subseteq \Gamma^{n}$ definable in the group $\Gamma$ with parameters from $\Gamma_{0}$. That is to say, the set $\Sigma_{n}$ is $\emptyset$-definable in the language $L_{t}$.

Using the theorem above, the following lemma follows immediately.

Lemma 2.2. Let $f$ and $g$ be automorphisms in Aut $\left(k / k_{0}\right)$ and Aut $\left(\Gamma / \Gamma_{0}\right)$ respectively. Then there is an automorphism of $k(\Gamma)$ in the pure field sense which extends both $f$ and $g$.

Proof. Define $\Sigma_{n}=\left\{\left(k_{1}, \ldots, k_{n}, \gamma_{1}, \ldots, \gamma_{n}\right): k_{1} \gamma_{1}+\cdots+k_{n} \gamma_{n}=0\right\} \subseteq k^{n} \times \Gamma^{n}$. By Theorem (2.1), the set $\Sigma_{n}$ is a finite union of sets $X \times Y$ with $X \subseteq k$ definable in the field $k$ with parameters from $k_{0}$ and $Y \subseteq \Gamma$ definable in the group $\Gamma$ with parameters from $\Gamma_{0}$. Therefore $\left(k_{1}, \ldots, k_{n}, \gamma_{1}, \ldots, \gamma_{n}\right) \in \Sigma_{n}$ if and only if $\left(f\left(k_{1}\right), \ldots, f\left(k_{n}\right), g\left(\gamma_{1}\right), \ldots, g\left(\gamma_{n}\right)\right) \in \Sigma_{n}$. This yields a well-defined ring automorphism $h$ of the ring $k[\Gamma]$ given by

$$
h\left(k_{1} \gamma_{1}+\cdots+k_{n} \gamma_{n}\right)=f\left(k_{1}\right) g\left(\gamma_{1}\right)+\cdots+f\left(k_{n}\right) g\left(\gamma_{n}\right)
$$

which further extends to the field $k(\Gamma)$. 
The following lemma (see [3]) will be crucial in order to characterize algebraic closure in the triple.

Lemma 2.3. [3, Lemma 2.1] Let $T_{1} \subset T_{2}$ be stable theories in the languages $L_{1}$ and $L_{2}$ respectively where $L_{1} \subseteq L_{2}$. Suppose that $T_{1}$ eliminates imaginaries. Let $M$ be a model of $T_{2}$ and $a, b$ be tuples in $M$. If $C$ is an algebraically closed set in the sense of $T_{2}$, then $a \underset{C}{\downarrow_{2}} b$ implies $a{\underset{C}{L}}_{L_{1}}^{T_{1}} b$.

Remark 2.4. Applying lemma (2.3), one sees that if $C$ is algebraically closed in the triple $(\Omega, k, \Gamma)$ and $a, b \in \Omega$, then the independence $a \underset{C}{t} b$ implies the algebraic independence $a \underset{C}{\downarrow} b$.

\section{Characterization of Algebraic Closure}

In this section, we give the characterization of algebraically closed structures in the triple which will be a key tool for all other proofs throughout the paper. In order to characterize algebraic closure, we depend upon the stability of the triple $(\Omega, k, \Gamma)$ which we know by $[5$, Proposition 8.3]. We begin with a definition.

Definition 3.1. We say that a substructure $A$ of the triple $(\Omega, k, \Gamma)$ is $(k, \Gamma)$-independent if

$$
A \underset{k_{A}\left(\Gamma_{A}\right)}{\stackrel{l d}{\downarrow}} k(\Gamma)
$$

Similarly $A$ is $k$-independent if $A \underset{k_{A}}{\downarrow} k$ and $A$ is $\Gamma$-independent if

$$
A \underset{\Gamma_{A}}{\downarrow} \Gamma
$$

Note that if $A$ is algebraically closed in the sense of the triple, then it is $k$-independent by [1, Remark 7.2].

Mann pairs over a subfield: We follow the notations of [5, Section 4, Page 7-8]. Let $\bar{a}=$ $\left(a_{1}, \ldots, a_{n}\right)$ be a tuple from $\Omega$. Define $\Sigma^{n d}(\bar{a}, k, \Gamma)$ as the set of all tuples $(\bar{b}, \bar{\gamma})=\left(b_{1}, \ldots, b_{n}, \gamma_{1}, \ldots, \gamma_{n}\right)$ where $b_{i}$ is in $k^{\times}$, the element $\gamma_{i}$ is in $\Gamma$ and $\left(\gamma_{1}, \ldots, \gamma_{n}\right)$ is a non-degenerate solution of $a_{1} b_{1} x_{1}+\cdots+a_{n} b_{n} x_{n}=0$. We let $\Sigma^{n d}(\bar{a}, k, \Gamma ; \bar{\gamma})$ be the set of all tuples $\bar{b} \in\left(k^{\times}\right)^{n}$ such that $(\bar{b}, \bar{\gamma}) \in \Sigma^{n d}(\bar{a}, k, \Gamma)$. Let $F$ be a subfield of $\Omega$. We say that $(k, \Gamma)$ is a Mann pair over $F$ if for every tuple $\bar{r}=\left(r_{1}, \ldots, r_{n}\right)$ from $F^{\times}, n \geq 2$, there is a finite subset $\Gamma(\bar{r})$ of $\Gamma$ such that

$$
\Sigma^{n d}(\bar{r}, k, \Gamma)=\bigcup_{\bar{\gamma} \in \Gamma(\bar{r})} \Sigma^{n d}(\bar{r}, k, \Gamma ; \bar{\gamma}) \times \Gamma \bar{\gamma}
$$

One can see that $(k, \Gamma)$ is a Mann pair over $k$ as it is a Mann pair. In [5, Proposition 4.7], it was proved that $(k, \Gamma)$ is a Mann pair over $\Omega$.

Lemma 3.2. Let $A$ be algebraically closed in the sense of the triple $(\Omega, k, \Gamma)$. Then $A$ is $(k, \Gamma)$-independent.

Proof. Since $A$ is also algebraically closed in the sense of $(\Omega, k)$, this yields that $A$ is $k$ independent, so $A \underset{k_{A}}{\stackrel{l d}{\perp}} k$ and therefore we have $A \underset{k_{A}\left(\Gamma_{A}\right)}{\stackrel{l d}{\perp}} k\left(\Gamma_{A}\right)$. By transitivity, it is enough to 
prove that

$$
A \underset{k\left(\Gamma_{A}\right)}{\stackrel{l d}{\downarrow}} k(\Gamma) .
$$

Suppose that $\alpha_{1}, \ldots, \alpha_{m}$ belong to $A$ and they are linearly dependent over $k(\Gamma)$. So there are elements $q_{1}, \ldots, q_{m}$ from $k(\Gamma)$ (not all zero) such that

$$
\alpha_{1} q_{1}+\cdots+\alpha_{m} q_{m}=0 .
$$

As each $q_{i}$ is of the form $\frac{u_{i}}{v_{i}}$ where $u_{i}, v_{i} \in k[\Gamma]$, by multiplying the equation (2) with the denominators, we see that $\alpha_{1}, \ldots, \alpha_{m}$ are linearly dependent over $k[\Gamma]$. Thus there are $t_{1}, \ldots, t_{m}$ from $k[\Gamma]$ such that

$$
\alpha_{1} t_{1}+\cdots+\alpha_{m} t_{m}=0 .
$$

Note that each $t_{i}$ is of the form $k_{i 1} g_{i 1}+\cdots+k_{i r} g_{i r}$ for some $r$. Therefore by equation (3), we get that there are elements $a_{1}, \ldots, a_{n}$ from $A^{\times}$(not necessarily distinct), $k_{1}, \ldots, k_{n}$ from $k^{\times}$and $g_{1}, \ldots, g_{n}$ from $\Gamma$ such that

$$
a_{1} k_{1} g_{1}+\cdots+a_{n} k_{n} g_{n}=0
$$

Without loss of generality, we may suppose that $\left(g_{1}, \ldots, g_{n}\right)$ is a non-degenerate solution of $a_{1} k_{1} x_{1}+\cdots+a_{n} k_{n} x_{n}=0$, because otherwise we split the equation into proper subsums such that all of them have the non-degeneracy condition for some subtuples of $\left(g_{1}, \ldots, g_{n}\right)$. In other words, we may assume that there is no non-trivial subsum of (4) which is zero. From (4), we see that

$$
1+\frac{a_{2}}{a_{1}} \frac{k_{2}}{k_{1}} \frac{g_{2}}{g_{1}}+\cdots+\frac{a_{n}}{a_{1}} \frac{k_{n}}{k_{1}} \frac{g_{n}}{g_{1}}=0 .
$$

Thus the tuple $\left(1, \frac{g_{2}}{g_{1}}, \ldots, \frac{g_{n}}{g_{1}}\right)$ is a non-degenerate solution of

$$
x_{1}+\frac{a_{2}}{a_{1}} \frac{k_{2}}{k_{1}} x_{2}+\cdots+\frac{a_{n}}{a_{1}} \frac{k_{n}}{k_{1}} x_{n}=0
$$

hence it is in $\Gamma \cdot \Gamma\left(1, \frac{a_{2}}{a_{1}}, \ldots, \frac{a_{n}}{a_{1}}\right)$ by $\left[5\right.$, Proposition 4.7]. So there are $\gamma \in \Gamma$ and $\left(\gamma_{1}, \ldots, \gamma_{n}\right) \in$ $\Gamma\left(1, \frac{a_{2}}{a_{1}}, \ldots, \frac{a_{n}}{a_{1}}\right)$ such that

$$
\left(1, \frac{g_{2}}{g_{1}}, \ldots, \frac{g_{n}}{g_{1}}\right)=\left(\gamma \gamma_{1}, \ldots, \gamma \gamma_{n}\right)
$$

This yields that

$$
\left(1, \frac{g_{2}}{g_{1}}, \ldots, \frac{g_{n}}{g_{1}}\right)=\left(1, \frac{\gamma_{2}}{\gamma_{1}}, \ldots, \frac{\gamma_{n}}{\gamma_{1}}\right)
$$

As $\Gamma\left(1, \frac{a_{2}}{a_{1}}, \ldots, \frac{a_{n}}{a_{1}}\right)$ is finite, we see that the equation (6) has only finitely many non-degenerate solutions in $\Gamma$, whose first coordinate is 1 . Since $A$ is algebraically closed in the triple, we see that the tuple $\left(1, \frac{g_{2}}{g_{1}}, \ldots, \frac{g_{n}}{g_{1}}\right)$ is in $A$. Now we finish the proof by dividing the equation (4) (or (3)) by $g_{1}$.

Remark 3.3. The proof of lemma (3.2) also shows that if $A$ is algebraically closed in the sense of the triple, then it is $\Gamma$-independent. This can also be proved directly by applying the Mann property, see [9, Lemma 2.4].

Next, we give the characterization of algebraic closure in the triple. 
Proposition 3.4. (Algebraic closure) Let $A \subset \Omega$ be a substructure in the sense of the triple. Then $A$ is algebraically closed in the sense of the triple if and only if $A$ and $k_{A}$ are algebraically closed fields, the group $\Gamma_{A}$ is algebraically closed in $\Gamma$ and $A$ is $(k, \Gamma)$-independent.

Proof. If $A$ is algebraically closed in the sense of the triple then $A$ and $k_{A}$ are algebraically closed fields and $\Gamma_{A}$ is algebraically closed in $\Gamma$. Moreover, by lemma (3.2) the structure $A$ is $(k, \Gamma)$-independent. Now we prove the converse. Let $\alpha$ be in $\Omega \backslash A$. Note that any field automorphism of $\Omega$ fixing $k$ and $\Gamma$ is an automorphism of the triple.

Case 1: If $\alpha \in \Gamma$. Then since $\Gamma_{A}$ is algebraically closed, we know that $\alpha$ has infinitely many conjugates in $\Gamma$. Choose a conjugate $\beta \in \Gamma$ of $\alpha$. Then there is an automorphism $f \in A u t\left(\Gamma / \Gamma_{A}\right)$ sending $\alpha$ to $\beta$. Since $\Gamma_{A}$ contains $\Gamma_{0}$, by lemma (2.2) there is an automorphism $h$ of $k(\Gamma)$ which is identity on $k$ and $f$ on $\Gamma$. Since $A$ is $(k, \Gamma)$-independent, by linear disjointness the former automorphism extends to a field automorphism of $A(k, \Gamma)$ over $A$ and this extends to an automorphism of $\Omega$ over $A$ which is actually an automorphism of the triple $(\Omega, k, \Gamma)$ over $A$. Thus $\alpha$ is not in $\operatorname{acl}_{t}(A)$. In particular, we have $\Gamma_{\operatorname{acl}_{t}(A)}=\Gamma_{A}$.

Case 2: If $\alpha \in k$. As $k_{A}$ is an algebraically closed field, we know that $\alpha$ has infinitely many conjugates in $k$. Choose a conjugate $\beta \in k$ of $\alpha$. Similar to the first case and by lemma (2.2) again, we construct an automorphism of the triple which sends $\alpha$ to $\beta$. Thus $\alpha$ is not in $\operatorname{acl}_{t}(A)$. This also indicates that $k_{\text {acl }_{t}(A)}=k_{A}$.

Case 3: If $\alpha \in \operatorname{acl}(A, k, \Gamma) \backslash A$. Then there exist $k_{1}, \ldots, k_{n} \in k$ and $g_{1}, \ldots, g_{n} \in \Gamma$ such that $\alpha \in \operatorname{acl}\left(A, k_{1}, \ldots, k_{n}, g_{1}, \ldots, g_{n}\right) \backslash A$. So for a rational polynomial

$$
r\left(x_{0}, x_{1}, \ldots, x_{n}, y_{1}, \ldots, y_{n}\right)
$$

with coefficients from $A$, we have that

$$
r\left(\alpha, k_{1}, \ldots, k_{n}, g_{1}, \ldots, g_{n}\right)=0 .
$$

Moreover, we may assume that $k_{1}, \ldots, k_{n}, g_{1}, \ldots, g_{n}$ are algebraically independent over $A$. By the first two cases, we know that $k_{i}$ and $g_{i}$ are not in $\operatorname{acl}_{t}(A)$ for $1 \leq i \leq n$. Thus the type

$$
p=\operatorname{tp}^{t}\left(k_{1}, \ldots, k_{n}, g_{1}, \ldots, g_{n} / \operatorname{acl}_{t}(A)\right)
$$

is non-algebraic. Now take $m_{1}, \ldots, m_{n}, h_{1}, \ldots, h_{n}$ such that

$$
\left(m_{1}, \ldots, m_{n}, h_{1}, \ldots, h_{n}\right) \models p
$$

and

$$
m_{1}, \ldots, m_{n}, h_{1}, \ldots, h_{n} \underset{\operatorname{acl}_{t}(A)}{\stackrel{t}{\perp}} k_{1}, \ldots, k_{n}, g_{1}, \ldots, g_{n} .
$$

By remark (2.4), we obtain the algebraic independence

$$
m_{1}, \ldots, m_{n}, h_{1}, \ldots, h_{n} \underset{\operatorname{acl}_{t}(A)}{\downarrow} k_{1}, \ldots, k_{n}, g_{1}, \ldots, g_{n} .
$$

We know that $\operatorname{acl}_{t}(A)$ is $(k, \Gamma)$-independent by lemma (3.2). As $k_{\text {acl }_{t}(A)}=k_{A}$ and $\Gamma_{\text {acl }_{t}(A)}=\Gamma_{A}$ from Case 1 and Case 2, by transitivity we get that

$$
m_{1}, \ldots, m_{n}, h_{1}, \ldots, h_{n} \underset{A}{\downarrow} k_{1}, \ldots, k_{n}, g_{1}, \ldots, g_{n} .
$$


Since there is a triple automorphism over $A$ sending $\left(k_{1}, \ldots, k_{n}, g_{1}, . ., g_{n}\right)$ to the tuple

$$
\left(m_{1}, \ldots, m_{n}, h_{1}, \ldots, h_{n}\right),
$$

this gives a conjugate $\beta$ of $\alpha$ with $\beta \in \operatorname{acl}\left(A, m_{1}, \ldots, m_{n}, h_{1}, \ldots, h_{n}\right)$ by the polynomial equation $r=0$. Observe that $\beta$ is different from $\alpha$ as we have

$$
m_{1}, \ldots, m_{n}, h_{1}, \ldots, h_{n} \underset{A}{\downarrow} k_{1}, \ldots, k_{n}, g_{1}, \ldots, g_{n}
$$

and $\alpha$ is not in $A$. Choosing other independent elements, as a result, we conclude that $\alpha$ has infinitely many conjugates over $A$ and hence $\alpha$ is not in $\operatorname{acl}_{t}(A)$.

Case 4: The element $\alpha$ is not in $\operatorname{acl}(A, k, \Gamma)$. Since any field automorphism of $\Omega$ fixing $k$ and $\Gamma$ is an automorphism of the triple, we deduce that $\operatorname{acl}(A, k, \Gamma)=\operatorname{acl}_{t}(A, k, \Gamma)$. This indicates that $\alpha$ is not in $\operatorname{acl}_{t}(A)$. Hence we are done.

Now we give several immediate corollaries of the previous proposition.

Corollary 3.5. For any subset $D$ in $\Omega$,

$$
\operatorname{acl}_{t}(D)=\operatorname{acl}\left(D, k_{\operatorname{acl}_{t}(D)}, \Gamma_{\operatorname{acl}_{t}(D)}\right) .
$$

If $B=\operatorname{acl}_{t}\left(k_{A}, \Gamma_{A}\right)$ where $A$ is algebraically closed in the sense of the triple, then $B=$ $\operatorname{acl}\left(k_{A}, \Gamma_{A}\right)$.

Proof. We put $E=\operatorname{acl}\left(D, k_{\operatorname{acl}_{t}(D)}, \Gamma_{\operatorname{acl}_{t}(D)}\right)$. As

$$
D \cup k_{\operatorname{acl}_{t}(D)} \cup \Gamma_{\operatorname{acl}_{t}(D)} \subseteq E \subseteq \operatorname{acl}_{t}(D),
$$

observe that $k_{E}=k_{\text {acl }_{t}(D)}$ and $\Gamma_{E}=\Gamma_{\text {acl }_{t}(D)}$. Since $\operatorname{acl}_{t}(D)$ is $(k, \Gamma)$-independent by lemma (3.2), we see that $E$ is also $(k, \Gamma)$-independent. We obtain by (3.4) that $E$ is algebraically closed in the sense of the triple. As $E$ contains $D$, we conclude that $E=\operatorname{acl}_{t}(D)$. For the second case, similarly $B=\operatorname{acl}_{t}\left(k_{A}, \Gamma_{A}\right) \subseteq A$ is $(k, \Gamma)$-independent and so we have that $F=\operatorname{acl}\left(k_{A}, \Gamma_{A}\right)$ and $k(\Gamma)$ are linearly disjoint over $k_{A}\left(\Gamma_{A}\right)$. In the proof of proposition (3.4), namely Case 1 and Case 2, we observe that $k_{F}=k_{A}$ and $\Gamma_{F}=\Gamma_{A}$. We finish the proof by proposition (3.4) again and hence $B=F$.

Corollary 3.6. Let $B$ be algebraically closed in the sense of the triple. Then $\Gamma_{B}=\Gamma_{\text {acl }_{t}(B, k)}$ and $k_{B}=k_{\text {acl }_{t}(B, \Gamma)}$. In particular, we have $B(k) \cap \Gamma=\Gamma_{B}$ and $B(\Gamma) \cap k=k_{B}$.

Proof. As $B$ is $(k, \Gamma)$-independent by lemma (3.2), we obtain that

$$
B(k) \underset{k\left(\Gamma_{B}\right)}{\stackrel{l d}{\perp}} k(\Gamma) \text { and } B(\Gamma) \underset{k_{B}(\Gamma)}{\stackrel{l d}{\perp}} k(\Gamma) .
$$

Therefore by proposition (3.4), we see that $\Gamma_{B}=\Gamma_{\operatorname{acl}_{t}(B, k)}$ and $k_{B}=k_{\text {acl }}(B, \Gamma)$. In particular, we obtain that $B(k) \cap \Gamma=\Gamma_{B}$ and $B(\Gamma) \cap k=k_{B}$.

Elementary substructure: We follow the notations of [6, Page 2]. Let $L$ be a subfield of $\Omega$, the integer $n \geq 2$ and $a_{1}, \ldots, a_{n}$ belong to $L^{\times}$. A solution $\left(s_{1}, \ldots, s_{n}\right) \in\left(\Omega^{\times}\right)^{n}$ of

$$
a_{1} x_{1}+\cdots+a_{n} x_{n}=0
$$


is called primitive over $L$ if it is linearly independent over $L$ for every non-empty proper subset $I$ of $\{1, \ldots, n\}$. Note that a primitive solution of (7) is also a non-degenerate solution of (7). For a subfield $k_{1}$ of $k$ and a subgroup $\Gamma_{1}$ of $\Gamma$, let $\Gamma_{1}\left(k_{1}, n\right)^{p r}$ be the set of all $\left(g_{1}, \ldots, g_{n}\right) \in \Gamma_{1}$ such that $g_{n}=1$ and $\left(g_{1}, \ldots, g_{n}\right)$ is a primitive solution of $(7)$ over $k_{1}$ for some tuple $\left(a_{1}, \ldots, a_{n}\right)$ from $k_{1}{ }^{\times}$. The following theorem is $[6$, Corollary 4.4] and the condition $(i)$ below is automatic as we assume that $k$ is a proper subfield of $\Omega$.

Theorem 3.7. $[6,4.4]$ Suppose that $\left(B, k_{B}, \Gamma_{B}\right) \subseteq(\Omega, k, \Gamma)$ is a substructure with $\left[B: k_{B}\right]>$ 2. Then $\left(B, k_{B}, \Gamma_{B}\right)$ is an elementary substructure of the triple if and only if the following conditions hold:

(i) $[\Omega: k]>2$,

(ii) $\Gamma(k, n)^{p r}=\Gamma_{B}\left(k_{B}, n\right)^{p r}$ for every $n \geq 2$,

(iii) $k_{B} \preceq k$ and $\Gamma_{B} \preceq \Gamma$,

(iv) $B \underset{k_{B}\left(\Gamma_{B}\right)}{\downarrow} k(\Gamma)$.

Using the previous result, we obtain the following corollary, which will also be used in section 6. Recall that a subgroup $\Gamma_{1}$ of $\Gamma$ is called pure if for every $c \in \Gamma_{1}$, the solvability of the equation $x^{n}=c$ in $\Gamma$ implies the solvability of the equation $x^{n}=c$ in $\Gamma_{1}$, where $n \geq 1$.

Corollary 3.8. Let $k_{1} \supseteq k_{0}$ and $\Gamma_{1} \supseteq \Gamma_{0}$ be algebraically closed substructures of $k$ and $\Gamma$ respectively. Then $B=\operatorname{acl}\left(k_{1}, \Gamma_{1}\right)$ is an algebraically closed substructure of $\Omega$ in the sense of the triple. Moreover, if $k_{1}$ and $\Gamma_{1}$ are elementary substructures of $k$ and $\Gamma$ respectively, then $B=\operatorname{acl}\left(k_{1}, \Gamma_{1}\right)$ is an elementary substructure of $\Omega$ in the sense of the triple.

Proof. Let $k_{1} \supseteq k_{0}$ and $\Gamma_{1} \supseteq \Gamma_{0}$ be algebraically closed substructures of $k$ and $\Gamma$ respectively and set $B=\operatorname{acl}\left(k_{1}, \Gamma_{1}\right)$. Observe that $\Gamma_{1}$ is a pure subgroup of $\Gamma$. As we have $\Gamma(k, n)^{p r}=\Gamma_{0}\left(k_{0}, n\right)^{p r}$ for every $n \geq 2$ and $k$ and $k_{0}(\Gamma)$ are linearly disjoint over $k_{0}$, we see that $\Gamma(k, n)^{p r}=\Gamma_{1}\left(k_{1}, n\right)^{p r}$ for every $n \geq 2$. Therefore, the field extension $k(\Gamma) \mid k_{1}\left(\Gamma_{1}\right)$ is regular by [6, Lemma 4.1]. In other words,

$$
B \underset{k_{1}\left(\Gamma_{1}\right)}{\stackrel{l d}{\downarrow}} k(\Gamma) .
$$

By proposition (3.4) and (8), we deduce that $B$ is algebraically closed in the sense of the triple. If $k_{1}$ and $\Gamma_{1}$ are elementary substructures of $k$ and $\Gamma$ respectively, then similar to the argument above, we conclude by Theorem (3.7) that $B$ is an elementary substructure of $\Omega$ in the sense of the triple, as most of the elements of $\Gamma_{0}$ are transcendental over $k$.

\section{Characterization of Forking}

In this section, we characterize forking in the triple. First, we need two lemmas. The following lemma states when two algebraically closed structures in the sense of the triple have the same type over a common substructure.

Lemma 4.1. Let $B_{1}, B_{2}$ and $C \subseteq B_{1} \cap B_{2}$ be three algebraically closed structures in the sense of the triple. Then $\operatorname{tp}^{t}\left(B_{1} / C\right)=\operatorname{tp}^{t}\left(B_{2} / C\right)$ if and only if there is a field automorphism over $C$ sending $B_{1}$ to $B_{2}$ with sending $k_{B_{1}}$ to $k_{B_{2}}$ and sending $\Gamma_{B_{1}}$ to $\Gamma_{B_{2}}$. 
Proof. Suppose that there is a field automorphism $\varphi$ over $C$ sending $B_{1}$ to $B_{2}$ with sending $k_{B_{1}}$ to $k_{B_{2}}$ and sending $\Gamma_{B_{1}}$ to $\Gamma_{B_{2}}$. In particular, we have $\operatorname{tp}_{k}\left(k_{B_{1}} / k_{C}\right)=\operatorname{tp}_{k}\left(k_{B_{2}} / k_{C}\right)$ and $\operatorname{tp}_{\Gamma}\left(\Gamma_{B_{1}} / \Gamma_{C}\right)=\operatorname{tp}_{\Gamma}\left(\Gamma_{B_{2}} / \Gamma_{C}\right)$. Thus, there is an automorphism $f \in A u t\left(k / k_{C}\right)$ sending $k_{B_{1}}$ to $k_{B_{2}}$ and there is an automorphism $g \in \operatorname{Aut}\left(\Gamma / \Gamma_{C}\right)$ sending $\Gamma_{B_{1}}$ to $\Gamma_{B_{2}}$. As $k_{C}$ contains $k_{0}$ and $\Gamma_{C}$ contains $\Gamma_{0}$, by lemma (2.2) there is an automorphism $h$ of $k(\Gamma)$ over $k_{C}\left(\Gamma_{C}\right)$ sending $k_{B_{1}}\left(\Gamma_{B_{1}}\right)$ to $k_{B_{2}}\left(\Gamma_{B_{2}}\right)$. Since $C$ is $(k, \Gamma)$-independent by lemma $(3.2)$, we have

$$
C \underset{k_{C}\left(\Gamma_{C}\right)}{\stackrel{l d}{\perp}} k(\Gamma),
$$

and the map $h$ further extends to an automorphism of $C(k, \Gamma)$ over $C$. Moreover, since each $B_{i}$ is $(k, \Gamma)$-independent by lemma $(3.2)$, we have that

$$
B_{i} \underset{k_{B_{i}}\left(\Gamma_{B_{i}}\right)}{\stackrel{l d}{L}} k(\Gamma) .
$$

Thus we get that

$$
B_{i} \underset{C\left(k_{B_{i}}, \Gamma_{B_{i}}\right)}{\stackrel{l d}{\perp}} C(k, \Gamma) .
$$

By the maps $\varphi$ and $h$, and the linear disjointness (9), we obtain an isomorphism $\tau$ between $B_{1}(k, \Gamma)$ and $B_{2}(k, \Gamma)$ over $C$. As $\tau$ extends to a field automorphism of $\Omega$ fixing $k$ and $\Gamma$ setwise, we conclude that $\operatorname{tp}^{t}\left(B_{1} / C\right)=\operatorname{tp}^{t}\left(B_{2} / C\right)$. The other direction is clear.

Now we prove another lemma before characterizing the independence in $(\Omega, k, \Gamma)$ and the idea partially comes from [2, Lemma 1.2].

Lemma 4.2. Let $C \subseteq A \cap B$ be three algebraically closed structures in the sense of the triple and suppose that $A \underset{C, k, \Gamma}{\downarrow} B, k, \Gamma$. Then $\operatorname{acl}_{t}(A, B)=\operatorname{acl}(A, B)$. Moreover, we have $k_{\text {acl }_{t}(A, B)}=$ $\operatorname{acl}\left(k_{A}, k_{B}\right)$ and $\Gamma_{\operatorname{acl}_{t}(A, B)}=\operatorname{acl}_{\Gamma}\left(\Gamma_{A}, \Gamma_{B}\right)$.

Proof. Since $A$ is algebraically closed, lemma (3.2) and transitivity yield that

$$
A \underset{C, k_{A}, \Gamma_{A}}{\perp} B, k, \Gamma
$$

and so $A \underset{B, k_{A}, \Gamma_{A}}{\downarrow} k, \Gamma$. As $B$ is algebraically closed, similarly we have that $B \underset{k_{B}, \Gamma_{B}}{\downarrow} k, \Gamma$ and thus

$$
B \underset{\operatorname{acl}\left(k_{A}, k_{B}\right)\left(\Gamma_{A} \Gamma_{B}\right)}{\downarrow} k, \Gamma
$$

By transitivity, we obtain that

$$
A, B \underset{\operatorname{acl}\left(k_{A}, k_{B}\right)\left(\Gamma_{A} \Gamma_{B}\right)}{\perp} k, \Gamma
$$

Note that $H=\operatorname{acl}_{\Gamma}\left(\Gamma_{A}, \Gamma_{B}\right) \subseteq \operatorname{acl}(A, B)$. Thus we have

$$
A, B \underset{\operatorname{acl}\left(k_{A}, k_{B}\right)(H)}{\downarrow} k, \Gamma \text {. }
$$

By corollary (3.8), we see that $\operatorname{acl}_{t}\left(k_{A}, k_{B}, H\right)=\operatorname{acl}\left(k_{A}, k_{B}, H\right)$ and in particular it is $(k, \Gamma)$ independent by lemma (3.2). By transitivity and in terms of linear disjointness we deduce 
that

$$
\operatorname{acl}(A, B) \underset{\operatorname{acl}\left(k_{A}, k_{B}\right)(H)}{\stackrel{l d}{\perp}} k(\Gamma) .
$$

Hence by proposition (3.4), we conclude that $\operatorname{acl}_{t}(A, B)=\operatorname{acl}(A, B)$ and also that $k_{\text {acl }_{t}(A, B)}=$ $\operatorname{acl}\left(k_{A}, k_{B}\right)$ and $\Gamma_{\operatorname{acl}_{t}(A, B)}=\operatorname{acl}_{\Gamma}\left(\Gamma_{A}, \Gamma_{B}\right)$.

Now we are ready to give the characterization of forking in the triple by applying proposition (3.4) and lemmas (4.1), (4.2). It turns out that independence in the triple is given by the algebraic independence in $\Omega$ and $k$; see $(i v)$ below. The proof of the following theorem is motivated from $[1,7.3]$.

Theorem 4.3. (Characterization of Forking)

Let $C=A \cap B$ and all of them be algebraically closed in the sense of the triple $T_{t}$. Then the following are equivalent:
(i) $A \underset{C}{\downarrow} B$
(ii) $A \underset{C, k, \Gamma}{\downarrow} B, k, \Gamma$ and $A \underset{C}{\downarrow} B$
(iii) $A \underset{C, k, \Gamma}{\downarrow} B, k, \Gamma$ and $k_{A}, \Gamma_{A} \underset{k_{C}, \Gamma_{C}}{\downarrow} k_{B}, \Gamma_{B}$
(iv) $A \underset{C, k, \Gamma}{\downarrow} B, k, \Gamma$ and $k_{A} \underset{k_{C}}{\downarrow} k_{B}$.

Proof. First suppose that $A \underset{C}{\downarrow} B$. By remark (2.4), we have that $A \underset{C}{\downarrow} B$. In particular, we obtain

$$
k_{A}, \Gamma_{A} \underset{C}{\downarrow} k_{B}, \Gamma_{B}
$$

Moreover, since $C$ is algebraically closed it is $(k, \Gamma)$-independent, thus we have that $C \underset{k_{C}, \Gamma_{C}}{\downarrow} k, \Gamma$ and in particular $C \underset{k_{C}, \Gamma_{C}}{\downarrow} k_{B}, \Gamma_{B}$. These two independence relations

$$
k_{A}, \Gamma_{A} \underset{C}{\downarrow} k_{B}, \Gamma_{B} \text { and } C \underset{k_{C}, \Gamma_{C}}{\downarrow} k_{B}, \Gamma_{B}
$$

give us that $k_{A}, \Gamma_{A} \underset{k_{C}, \Gamma_{C}}{\downarrow} k_{B}, \Gamma_{B}$ by transitivity. Similarly, the independence $A \underset{C}{\downarrow} B$ yields that $k_{A} \underset{C}{\downarrow} k_{B}$. Moreover, as $C$ is algebraically closed in the pair $(\Omega, k)$ it is $k$-independent. Thus we have $C \underset{k_{C}}{\downarrow} k$ and so $C \underset{k_{C}}{\downarrow} k_{B}$. By transitivity we deduce that $k_{A} \underset{k_{C}}{\downarrow} k_{B}$.

Now we prove that $A \underset{C, k, \Gamma}{\downarrow} B, k, \Gamma$. Suppose for a contradiction that

$$
A \underset{C, k, \Gamma}{\lfloor} B, k, \Gamma
$$

Let $q=\operatorname{tp}\left(B / C \cup k_{B} \cup \Gamma_{B}\right)$ and $\lambda \geq \omega_{1}$. As $B$ is algebraically closed and contains $C$, by lemma (3.2) we get that

$$
B \underset{C, k_{B}, \Gamma_{B}}{\downarrow} C, k, \Gamma .
$$


Thus by saturation, there exists $\left(B_{i}\right)_{i \leq \lambda}$ with $B=B_{0}$ such that $B_{i} \models q$ and $\left(B_{i}\right)_{i \leq \lambda}$ is independent over $C \cup k \cup \Gamma$ in the field sense, and also $B_{i} \underset{C, k_{B}, \Gamma_{B}}{\downarrow} C, k, \Gamma$ by (10). By the choice of $\left(B_{i}\right)_{i \leq \lambda}$, we get that $k_{B} \subseteq k_{B_{i}}$ and $\Gamma_{B} \subseteq \Gamma_{B_{i}}$ for all $i$. On the other hand, by the independence $B_{i} \downarrow C, k, \Gamma$ we have that $k_{B_{i}}, \Gamma_{B_{i}} \subseteq \operatorname{acl}\left(C, k_{B}, \Gamma_{B}\right) \subseteq B$. Thus we obtain the equalities $C, k_{B}, \Gamma_{B}$

$k_{B}=k_{B_{i}}$ and $\Gamma_{B}=\Gamma_{B_{i}}$ for all $i$. As acl $\left(C, k_{B}, \Gamma_{B}\right) \subseteq B$, this yields that $k_{B}=k_{\text {acl }\left(C, k_{B}, \Gamma_{B}\right)}$ and $\Gamma_{B}=\Gamma_{\operatorname{acl}\left(C, k_{B}, \Gamma_{B}\right)}$. Since $B$ is $(k, \Gamma)$-independent by lemma $(3.2)$, in particular we see that

$$
\operatorname{acl}\left(C, k_{B}, \Gamma_{B}\right) \underset{k_{B}\left(\Gamma_{B}\right)}{\stackrel{l d}{\downarrow}} k(\Gamma) .
$$

As $B_{i} \underset{C, k_{B}, \Gamma_{B}}{\downarrow} k, \Gamma$, by transitivity and in terms of linear disjointness, we obtain that

$$
B_{i} \underbrace{l d}_{k_{B}\left(\Gamma_{B}\right)} k(\Gamma) .
$$

Therefore by proposition (3.4), we deduce that $B_{i}$ is algebraically closed in the sense of the triple for all $i$. Then, lemma (4.1) yields that $\operatorname{tp}^{t}\left(B_{i} / C\right)=\operatorname{tp}^{t}(B / C)$ for all $i$. By Erdős-Rado Theorem, we may assume that $\left(B_{i}\right)_{i \leq \lambda}$ is $C$-indiscernible in the sense of $T_{t}$. Let $p_{i}$ be the pullback of the type $\operatorname{tp}^{t}(A / B)$ under $B_{i} \longrightarrow B$. Since $A \underset{C}{\downarrow} B$, we know that $\bigcup_{i \leq \lambda} p_{i}\left(x, B_{i}\right)$ is consistent. So there exists $A_{1}$ such that $\operatorname{tp}^{t}\left(A_{1} B_{i}\right)=\operatorname{tp}^{t}(A B)$ for all $i$. Now $\left(B_{i}\right)_{i \leq \lambda}$ is independent over $C \cup k \cup \Gamma$ and $A_{1} \underset{C, k, \Gamma}{\lfloor} B_{i}$ for each $B_{i}$. This contradicts the stability of the field $\Omega$. Hence we proved that $(i)$ implies $(i i),(i i i)$ and $(i v)$.

Now we prove that $(i i),(i i i)$ and $(i v)$ are equivalent. We already proved that $(i i)$ implies $(i i i)$ and $(i v)$ in the beginning. First, we make the following observation. Since $A$ is algebraically closed in the sense of the triple, it is $(k, \Gamma)$-independent by lemma (3.2). In particular, one has $A \underset{C, k_{A}, \Gamma_{A}}{\downarrow} C, k, \Gamma$. Thus if we have $A \underset{C, k, \Gamma}{\downarrow} B$, this yields by transitivity that

$$
A \underset{C, k_{A}, \Gamma_{A}}{\downarrow} B
$$

Suppose that we have $(i i i)$ and we will show that $(i i)$ holds. Note that as $A$ is $(k, \Gamma)$-independent we also have that $C\left(k_{A}, \Gamma_{A}\right)$ is $(k, \Gamma)$-independent and in particular

$$
C\left(k_{A}, \Gamma_{A}\right) \underset{k_{A}, \Gamma_{A}}{\downarrow} k_{B}, \Gamma_{B}
$$

As we also have

$$
k_{A}, \Gamma_{A} \underset{k_{C}, \Gamma_{C}}{\downarrow} k_{B}, \Gamma_{B}
$$

by transitivity we obtain that $C\left(k_{A}, \Gamma_{A}\right) \underset{k_{C}, \Gamma_{C}}{\downarrow} k_{B}, \Gamma_{B}$ and hence $k_{A}, \Gamma_{A} \underset{C}{\downarrow} k_{B}, \Gamma_{B}$. As $B$ is $(k, \Gamma)$-independent, we deduce the independence $k_{A}, \Gamma_{A} \underset{C}{\downarrow} B$ by transitivity. From the previous independence and by (11), we conclude that $A \underset{C}{\downarrow} B$ by transitivity again. Thus (iii) implies (ii). Now assume that we have $(i v)$ and we show $(i i)$. As $A$ is algebraically closed in the pair $(\Omega, k)$, 
it is $k$-independent. In particular, we have $C, k_{A} \underset{k_{A}}{\downarrow} k_{B}$. By $k_{A} \underset{k_{C}}{\downarrow} k_{B}$ and transitivity, we obtain that $k_{A} \underset{C}{\downarrow} k_{B}$. As $B$ is also $k$-independent, we see that $B \underset{k_{B}}{\downarrow} k_{A}$. By the previous independence and $k_{A} \underset{C}{\downarrow} k_{B}$, we deduce that $B \underset{C}{\downarrow} k_{A}$ by transitivity. Next, we prove that $B \underset{C, k_{A}}{\downarrow} \Gamma_{A}$. So let $b_{1}, \ldots, b_{n}$ be in $B$, the elements $k_{1}, \ldots, k_{n}$ be in $k_{A}$ and $g_{1}, \ldots, g_{n}$ be in $\Gamma_{A}$ such that

$$
b_{1} k_{1} g_{1}+\cdots+b_{n} k_{n} g_{n}=0
$$

We may suppose that no proper subsum of this equation is zero. Since $\Gamma$ has the Mann property over $\Omega$, we obtain that $\frac{g_{i}}{g_{1}} \in \operatorname{acl}_{t}\left(B, k_{A}\right)$ and so $\frac{g_{i}}{g_{1}} \in \Gamma_{B}$ for all $i$ by lemma (3.6). As $\frac{g_{i}}{g_{1}} \in \Gamma_{A}$ also, we obtain that $\frac{g_{i}}{g_{1}} \in \Gamma_{C}$ for all $i$. Thus we have $B \underset{C, k_{A}}{\downarrow} \Gamma_{A}$. By $B \underset{C, k_{A}}{\downarrow} \Gamma_{A}$ and $B \underset{C}{\downarrow} k_{A}$, we obtain that $B \underset{C}{\downarrow} k_{A}, \Gamma_{A}$. The previous independence and (11) give again that $A \underset{C}{\downarrow} B$.

Lastly, we prove that $(i i)$ implies $(i)$. Our strategy for the proof of non-forking is to show consistency of conjugates of types along a Morley sequence; see [21, Proposition 7.2.14]. So let $\lambda$ be an uncountable cardinal and $\left(B_{i}\right)_{i<\lambda}$ be a Morley sequence over $C$ in the sense of the triple where $B_{0}=B$. Note that $\left(B_{i}, k_{B_{i}}, \Gamma_{B_{i}}\right)_{i<\lambda}$ is also a Morley sequence over $C$ in the sense of the triple but for simplicity we write $\left(B_{i}\right)_{i}$ instead. Next we may assume the sequence $\left(B_{i}\right)_{i}$ is a Morley sequence over $C k_{A} \Gamma_{A}$ in the field sense, as it can be seen from the following argument: We observe by remark (2.4) that $\left(B_{i}\right)_{i}$ is $C$-independent in the field sense. By $(i i)$ we also have that

$$
k_{A}, \Gamma_{A} \underset{C}{\downarrow} B
$$

By stability, we cannot have that

$$
k_{A}, \Gamma_{A} \underset{C}{\lfloor} B_{i}
$$

for many $i$, as $\left(B_{i}\right)_{i}$ is $C$-independent in the field sense. So we may assume that

$$
k_{A}, \Gamma_{A} \underset{C}{\downarrow} B_{i}
$$

for all $i$. Since $C$ is an algebraically closed field and types over algebraically closed subfields are stationary in the pure field $\Omega$, by (12) and (13) we deduce that $\operatorname{tp}\left(B_{i} / C k_{A} \Gamma_{A}\right)=\operatorname{tp}\left(B / C k_{A} \Gamma_{A}\right)$ for all $i$. Now by Erdős-Rado Theorem, we may assume that $\left(B_{i}\right)_{i}$ is $C k_{A} \Gamma_{A}$-indiscernible in the sense of the pure field $\Omega$. By [21, Lemma 7.2.19], we get that

$$
k_{A}, \Gamma_{A} \underset{C}{\downarrow}\left\{B_{i}: i<\lambda\right\} .
$$

By transitivity and as $\left(B_{i}\right)_{i}$ is $C$-independent, we conclude that $\left(B_{i}\right)_{i}$ is a Morley sequence over $C k_{A} \Gamma_{A}$ in the field sense.

Since $A \underset{C}{\downarrow} B$, we also have

$$
A \underset{C, k_{A}, \Gamma_{A}}{\downarrow} B, k_{A}, \Gamma_{A} .
$$


Let $p(x)=\operatorname{tp}\left(A / B \cup k_{A} \cup \Gamma_{A}\right)$ and $p_{i}(x)$ be the copy over $B_{i} \cup k_{A} \cup \Gamma_{A}$. Thus there exists a long tuple $D$ such that $D \models \bigcup_{i} p_{i}(x)$. Moreover as all $B_{i}, k$ and $\Gamma$ are small in $\Omega$, by taking a non-forking extension we may assume that

$$
D \underset{B, k_{A}, \Gamma_{A}}{\perp}\left\{B_{i}: i<\lambda\right\}, k, \Gamma .
$$

As $A \underset{C, k_{A}, \Gamma_{A}}{\downarrow} B, k_{A}, \Gamma_{A}$, by transitivity we see that

$$
D \underset{C, k_{A}, \Gamma_{A}}{\downarrow} B_{i}, k, \Gamma
$$

for all $i$. Observe that $k_{D}=k_{A}$ and $\Gamma_{D}=\Gamma_{A}$ as $D$ and $A$ has the same type over $B k_{A} \Gamma_{A}$ and by (15). Moreover, there is a field automorphism $f_{i}$ of $\Omega$ over $C k_{A} \Gamma_{A}$ sending $A B k_{B} \Gamma_{B}$ to $D B_{i} k_{B_{i}} \Gamma_{B_{i}}$. Since $A$ is $(k, \Gamma)$-independent and $C \subseteq A$, we also see that

$$
\operatorname{acl}\left(C, k_{A}, \Gamma_{A}\right) \underset{k_{A}\left(\Gamma_{A}\right)}{\stackrel{l d}{\perp}} k(\Gamma) .
$$

Since also we have $D \underset{C, k_{A}, \Gamma_{A}}{\downarrow} k, \Gamma$, by transitivity and in terms of linear disjointness, we deduce that

$$
D \underset{k_{A}\left(\Gamma_{A}\right)}{\stackrel{l d}{\perp}} k(\Gamma) .
$$

Therefore by proposition (3.4), we conclude that $D$ is algebraically closed in the sense of the triple. By lemma (4.2), we see that $\operatorname{acl}_{t}(A, B)=\operatorname{acl}(A, B)$, and also $k_{\text {acl }_{t}(A, B)}=\operatorname{acl}\left(k_{A}, k_{B}\right)$ and $\Gamma_{\operatorname{acl}_{t}(A, B)}=\operatorname{acl}_{\Gamma}\left(\Gamma_{A}, \Gamma_{B}\right)$. Hence, we have

$$
\operatorname{acl}(A, B) \underset{\operatorname{acl}\left(k_{A}, k_{B}\right)\left(\operatorname{acl}_{\Gamma}\left(\Gamma_{A}, \Gamma_{B}\right)\right)}{l d} k(\Gamma) .
$$

By the choice of $D$ and by lemma (4.2) again, we also have that

$$
\operatorname{acl}\left(D, B_{i}\right) \underbrace{\stackrel{l d}{\perp}\left(\operatorname{acl}_{\Gamma}\left(\Gamma_{A}, \Gamma_{B_{i}}\right)\right)}_{\operatorname{acl}\left(k_{A}, k_{B_{i}}\right)} k(\Gamma) .
$$

As $\left(B_{i}\right)_{i}=\left(B_{i}, k_{B_{i}}, \Gamma_{B_{i}}\right)$ is a Morley sequence over $C k_{A} \Gamma_{A}$ in the field sense, there is a partial field isomorphism $\sigma_{i}$ of $\Omega$ over $C k_{A} \Gamma_{A}$ sending $B$ to $B_{i}$, with $k_{B}$ to $k_{B_{i}}$ and $\Gamma_{B}$ to $\Gamma_{B_{i}}$. In particular, there is a partial field isomorphism $g_{i}$ sending $k_{B}$ to $k_{B_{i}}$ over $k_{A}$, and there is a partial group isomorphism $h_{i}$ sending $\Gamma_{B}$ to $\Gamma_{B_{i}}$ over $\Gamma_{A}$. By strong homogeneity and (2.2), there is a field automorphism $\psi_{i}$ of $k(\Gamma)$ (obtained by $g_{i}$ and $\left.h_{i}\right) \operatorname{sending} \operatorname{acl}\left(k_{A}, k_{B}\right)\left(\operatorname{acl} \Gamma_{\Gamma}\left(\Gamma_{A}, \Gamma_{B}\right)\right.$ ) to $\operatorname{acl}\left(k_{A}, k_{B_{i}}\right)\left(\operatorname{acl}_{\Gamma}\left(\Gamma_{A}, \Gamma_{B_{i}}\right)\right)$. Now by the two linear disjointness (16) and (17), we obtain a partial field isomorphism $\tau_{i}$ preserving $k, \Gamma$ and sending $\operatorname{acl}(A B)$ to $\operatorname{acl}\left(D B_{i}\right)$. As $\tau_{i}$ extends to an automorphism of $\Omega$, which is indeed an automorphism of the triple, we conclude that $\operatorname{tp}^{t}\left(D B_{i} k_{A} \Gamma_{A}\right)=\operatorname{tp}^{t}\left(A B k_{A} \Gamma_{A}\right)$ for all $i$. Hence we have $(i)$.

Remark 4.4. The proof of Theorem (4.3) above indicates the characterization of independence in pairs $(\Omega, k)$ and $(\Omega, \Gamma)$. More precisely, let $C=A \cap B$ and all of them be algebraically closed in the pair $(\Omega, k)$. Then $A$ and $B$ are independent over $C$ in $(\Omega, k)$ if and only if $A \underset{C, k}{\downarrow} B, k$ and 
$k_{A} \downarrow k_{B}$. If $C=A \cap B$ and all are algebraically closed in the pair $(\Omega, \Gamma)$, then $A$ and $B$ are

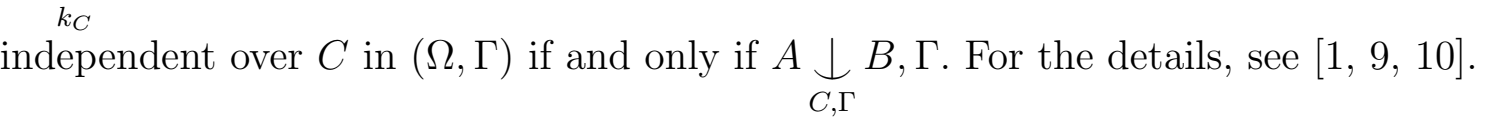

Corollary 4.5. For every $a \in \Omega$, we have that

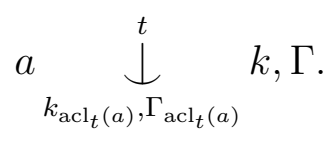

Moreover, we also have the independence $a \underset{\Gamma_{\mathrm{acl}_{t}(a)}^{\perp}}{\perp} \Gamma$.

Proof. Since $\operatorname{acl}_{t}(a)$ is $(k, \Gamma)$-independent, we have that

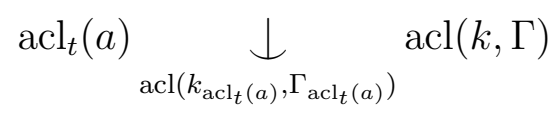

and $\operatorname{acl}_{t}(a) \cap \operatorname{acl}(k, \Gamma)=\operatorname{acl}\left(k_{\operatorname{acl}_{t}(a)}, \Gamma_{\operatorname{acl}_{t}(a)}\right)$. By corollary (3.5), we see that

$$
\operatorname{acl}\left(k_{\operatorname{acl}_{t}(a)}, \Gamma_{\operatorname{acl}_{t}(a)}\right)=\operatorname{acl}_{t}\left(k_{\operatorname{acl}_{t}(a)}, \Gamma_{\operatorname{acl}_{t}(a)}\right)
$$

and also that $\operatorname{acl}_{t}(k, \Gamma)=\operatorname{acl}(k, \Gamma)$. Therefore, we deduce that

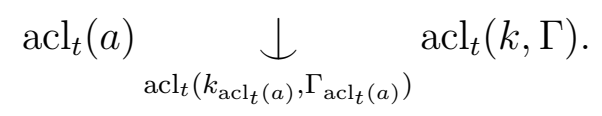

Applying Theorem (4.3), we obtain the first part. For the second part, we have $\operatorname{acl}_{t}(\Gamma)=$ $\operatorname{acl}\left(\Gamma, k_{0}\right)$ and $\operatorname{acl}_{t}\left(\Gamma_{\operatorname{acl}_{t}(a)}\right)=\operatorname{acl}\left(\Gamma_{\operatorname{acl}_{t}(a)}, k_{0}\right)$, and also that $k_{\operatorname{acl}_{t}(\Gamma)}=k_{0}$ by corollary (3.8). As $\operatorname{acl}_{t}(a)$ is $\Gamma$-independent by remark (3.3), we conclude by Theorem (4.3) similar to the first part.

The next lemma states that the independence in the triple implies the independence in $\Gamma$ as a pure group.

Lemma 4.6. Let $C=A \cap B$ and all of them be algebraically closed in the sense of the triple and $A \underset{C}{t} B$. Then we have the independence $\Gamma_{A} \underset{\Gamma_{C}}{\stackrel{\Gamma}{\downarrow}} \Gamma_{B}$ in the abelian group $\Gamma$.

Proof. As $A \underset{C}{\downarrow}{\underset{L}{t}}^{t} B$, we have $\Gamma_{A}{\underset{C}{t}}_{t}^{t} \Gamma_{B}$. Corollary (4.5) and the transitivity of the independence yield that $\Gamma_{A} \underset{\Gamma_{C}}{\downarrow} \Gamma_{B}$. Hence we conclude that $\Gamma_{A} \underset{\Gamma_{C}}{\stackrel{\Gamma}{\downarrow}} \Gamma_{B}$.

4.1. Independence over Models and Reducts. In this subsection we study the independence over models. Then we investigate the relation between the independence in the triple and the independence in $(\Omega, k)$ and $(\Omega, \Gamma)$.

Proposition 4.7. Let $M=A \cap B$ where $A, B$ are algebraically closed in the sense of the triple $T_{t}$ and $M$ is a model of $T_{t}$. Then $A \underset{M}{\stackrel{t}{\perp}} B$ if and only if $A \underset{M\left(k_{A}, \Gamma_{A}\right)}{\stackrel{l d}{\perp}} B(k, \Gamma)$ and $k_{A} \underset{k_{M}}{\downarrow} k_{B}$.

Proof. By Theorem (4.3)(iv), it is enough to prove that $A{\underset{M}{\downarrow}}^{t} B \operatorname{implies} A \underset{M\left(k_{A}, \Gamma_{A}\right)}{\downarrow} B(k, \Gamma)$. Now let $a_{1}, \ldots, a_{n}$ be in $A$ (not necessarily distinct), the elements $b_{1}, \ldots, b_{n}$ be in $B$, the elements 
$k_{1}, \ldots, k_{n}$ be in $k$ and $g_{1}, \ldots, g_{n}$ be in $\Gamma$ such that

$$
a_{1} b_{1} k_{1} g_{1}+\cdots+a_{n} b_{n} k_{n} g_{n}=0 .
$$

Suppose that $f(\bar{x}, \bar{y}, \bar{z}, \bar{t})$ is the formula $x_{1} y_{1} z_{1} t_{1}+\cdots+x_{n} y_{n} z_{n} t_{n}=0$. Let $\phi(\bar{x}, \bar{y})$ be the formula

$$
\exists \bar{z} \in P_{1} \exists \bar{t} \in P_{2} f(\bar{x}, \bar{y}, \bar{z}, \bar{t}) .
$$

Then $\bar{a}=\phi(\bar{x}, \bar{b})$. As $A \underset{M}{\downarrow} B$, by stability the type $\operatorname{tp}^{t}(A / B)$ is an heir extension of $\operatorname{tp}^{t}(A / M)$. So there is $\bar{m} \in M$ such that $\bar{a} \models \phi(\bar{x}, \bar{m})$. We finish the proof by transitivity as $A$ is $(k, \Gamma)$ independent.

Proposition 4.8. Let $C=A \cap B$ and all of them be algebraically closed in the sense of the triple $T_{t}$. If $A \underset{C}{\downarrow} B$ then we have $A \underset{C}{\perp_{1}} B$ and $A \underset{C}{P_{2}} B$ in the sense of the pairs $(\Omega, k)$ and $(\Omega, \Gamma)$ respectively.

Proof. Suppose that $A \underset{C}{\stackrel{t}{\downarrow}} B$. By Theorem (4.3)(iv), we know that $A \underset{C, k, \Gamma}{\downarrow} B, k, \Gamma$ and $k_{A} \underset{k_{C}}{\downarrow} k_{B}$. As $A$ is $(k, \Gamma)$-independent by lemma (3.2), transitivity yields that $A \downarrow \downarrow \quad B, k, \Gamma$. Thus by remark (4.4), it is enough to show that $A \underset{C, k}{\downarrow} B, k$ and $A \underset{C, \Gamma}{\downarrow} B, \Gamma$. Note that by corollary (3.6), we have that $\Gamma_{\operatorname{acl}_{t}(B, k)}=\Gamma_{B}$ and $k_{\operatorname{acl}_{t}(B, \Gamma)}=k_{B}$. In order to show $A \underset{C, k}{\downarrow} B, k$, by transitivity it is enough to show that $B(k) \underset{C(k)}{\stackrel{l d}{\downarrow}} C\left(k, \Gamma_{A}\right)$. So let $b_{1}, \ldots, b_{n}$ be in $B$, the elements $k_{1}, \ldots, k_{n}$ be in $k$ and $g_{1}, \ldots, g_{n}$ be in $\Gamma_{A}$ such that

$$
b_{1} k_{1} g_{1}+\cdots+b_{n} k_{n} g_{n}=0 .
$$

We may suppose that no proper subsum of this equation is zero. Since $\Gamma$ has the Mann property over $\Omega$, we obtain that $\frac{g_{i}}{g_{1}} \in \operatorname{acl}_{t}(B, k)$ and so $\frac{g_{i}}{g_{1}} \in \Gamma_{B}$ for all $i$. As $\frac{g_{i}}{g_{1}} \in \Gamma_{A}$ also, we obtain that $\frac{g_{i}}{g_{1}} \in \Gamma_{C}$ for all $i$. Thus we have what we desired. Similarly, to prove $A \underset{C, \Gamma}{\downarrow} B, \Gamma$, we need to show that $B(\Gamma) \underset{C(\Gamma)}{\downarrow} k_{A}$. Since $k_{\text {acl }_{t}(B, \Gamma)}=k_{B}$ and $\operatorname{acl}_{t}(B, \Gamma)$ is $k$-independent, in particular we obtain that $B(\Gamma) \underset{k_{B}}{\downarrow} k_{A}$. As we also have $k_{B} \underset{k_{C}}{\downarrow} k_{A}$, we conclude by transitivity. Hence we have the proposition.

Now we give more equivalences for the characterization of forking in the triple:

Corollary 4.9. Let $C=A \cap B$ and all of them be algebraically closed in the sense of the triple $T_{t}$. Then the following are equivalent:
(i) $A \underset{C}{\downarrow} B$,
(ii) $A \underset{C, k, \Gamma}{\stackrel{C}{\downarrow}} B, k, \Gamma$ and $A \underset{C}{\downarrow} B$
(iii) $A \underset{C, k, \Gamma}{\downarrow} B, k, \Gamma$ and $k_{A}, \Gamma_{A} \underset{k_{C}, \Gamma_{C}}{\downarrow} k_{B}, \Gamma_{B}$,
(iv) $A \underset{C, k, \Gamma}{\downarrow} B, k, \Gamma$ and $k_{A} \underset{k_{C}}{\downarrow} k_{B}$. 


\section{(v) $A \underset{C, k, \Gamma}{\downarrow} B, k, \Gamma$ and $A \underset{C, \Gamma}{\downarrow} B, \Gamma$.}

Proof. By Theorem (4.3) and proposition (4.8) we know that (i), (ii), (iii) and (iv) are equivalent and $(i)$ implies $(v)$. Now we show that $(v)$ implies $(i i)$. If we have $A \underset{C, \Gamma}{\downarrow} B, \Gamma$, then lemma (2.3) and remark (4.4) yield the desired independence $A \underset{C}{\downarrow} B$ since the independence $A \underset{C, \Gamma}{\downarrow} B, \Gamma$ implies $A \underset{C}{\stackrel{P_{2}}{\downarrow}} B$.

Remark 4.10. Note that in corollary $(4.9)(\mathrm{v})$, we cannot replace $A \underset{C, \Gamma}{\downarrow} B, \Gamma$ by $A \underset{C, k}{\downarrow} B, k$ since the latter independence does not imply the independence $A \underset{C}{\downarrow} B$.

\section{Definable Groups}

In this section, we characterize definable groups in the triple $(\Omega, k, \Gamma)$ up to isogeny. It emerges that definable groups in the triple are given by definable and interpretable groups in $\Omega, k$ and $\Gamma$. The following lemma is from [19]:

Lemma 5.1. $[19,5.4]$ Suppose that $G$ is a stable group. Every formula $\varphi(x, y)$ can be associated with a natural number $n=n(\varphi)$ such that, if $A$ is a generic subset of $G$ defined by a formula $\varphi(x, a)$, then $G$ is covered by $n$ translates of $A$.

The next lemma affirms when a type-definable group in the triple is actually an algebraic group and our method is similar to the result [2, Lemma 2.1].

Lemma 5.2. Let $H$ be a connected $T_{t}$-type-definable subgroup of an algebraic group $V$, all definable over an algebraically closed set $A$ in the sense of the triple. Let a be the generic over $A$ which lies in some translate of $H$ which is also definable over $A$. If $k_{\operatorname{acl}_{t}(a, A)}=k_{A}$ and $\Gamma_{\text {acl }_{t}(a, A)}=\Gamma_{A}$, then $H$ is an algebraic group. In particular $H$ is definable.

Proof. First we may assume that $a \in H$ : Suppose that $a \in b H$. Let $a^{\prime}$ be such that $\operatorname{tp}^{t}\left(a^{\prime} / A\right)=$ $\operatorname{tp}^{t}(a / A)$ and $a^{\prime}{\underset{A}{t}}_{\perp}^{t} a$. Put $a_{1}=a^{-1} a^{\prime}$. Then we have $a_{1} \underset{A}{\downarrow_{A}^{t}} a$ and $a_{1} \in H$ is generic. Since $a^{\prime}{\underset{A}{t}}_{A}^{t} a$, we have that $a^{\prime}, A{\underset{A}{t}}_{t}^{t} a, A$. So by lemma (4.2) and Theorem (4.3), we see that

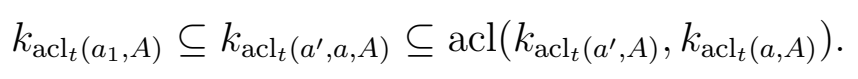

Since $k_{\text {acl }_{t}\left(a^{\prime}, A\right)}=k_{\operatorname{acl}_{t}(a, A)}=k_{A}$, we deduce that $k_{\operatorname{acl}_{t}\left(a_{1}, A\right)}=k_{\operatorname{acl}_{t}(A)}$. Similarly we obtain that $\Gamma_{\operatorname{acl}_{t}\left(a_{1}, A\right)}=\Gamma_{A}$. Thus we may assume that $a \in H$ by changing $a$ with $a_{1}$. Put $p=\operatorname{tp}^{t}(a / A)$ and $p_{0}$ its $T$-reduct where $T$ is the theory of the field $\Omega$. Let $H_{0}$ be the smallest algebraic group containing $H$ which exists by the assumption and the $\omega$-stability of $\Omega$. Note that $H=\operatorname{stab}_{t}(p) \subset \operatorname{stab}_{T}\left(p_{0}\right)$. So $H_{0} \subseteq \operatorname{stab}_{T}\left(p_{0}\right)$. On the other hand, since $p_{0}(x)$ implies that $x \in H_{0}$, we get that $\operatorname{stab}_{T}\left(p_{0}\right) \subseteq H_{0}$. Thus we have the equality and moreover $H_{0}$ is $T$-connected. To prove the lemma it is enough to show that $p$ is the unique generic of $H_{0}$ in the sense of the triple, as this implies that $H_{0}=\operatorname{stab}_{t}(p)=H$. Let $h$ be a generic of $H_{0}$ over $A$ in the sense of the triple and put $q=\operatorname{tp}^{t}(h / A)$. Note that $h$ is in particular a generic in the sense of the field 
so it has the same field type over $A$

Claim: We have $h \downarrow k, \Gamma$.

Proof of the Claim: First note that $a \in H_{0}$. As the algebraic closure $\operatorname{acl}_{t}(a, A)$ is $(k, \Gamma)$ independent by lemma (3.2) and by the assumptions $k_{\text {acl }_{t}(a, A)}=k_{A}$ and $\Gamma_{\text {acl }_{t}(a, A)}=\Gamma_{A}$, we have that $a, A \underset{k_{A}, \Gamma_{A}}{\downarrow} k, \Gamma$ and so $a \underset{A}{\downarrow} k, \Gamma$. As a consequence, we see that $a$ is a generic of $H_{0}$ over $A \cup k \cup \Gamma$ in the sense of the field $\Omega$. Now if $h \underset{A}{\not \ell} k, \Gamma$, then there exists a formula $\varphi(x, m, \gamma) \in$ $\operatorname{tp}(h / A, k, \Gamma)$ which is not generic in $H_{0}$, with parameters from $A$, where $m \in k$ and $\gamma \in \Gamma$. Put $n=n(\varphi)$ as in lemma (5.1) and

$$
\theta(y, z)=\exists h_{1} \ldots \exists h_{n} \in H_{0}\left(\forall x \in H_{0} \bigvee_{i \leq n} \varphi\left(h_{i}{ }^{-1} x, h_{i}{ }^{-1} y, h_{i}{ }^{-1} z\right)\right)
$$

and $\phi(x, y, z)=\neg \theta(y, z) \wedge \varphi(x, y, z)$. Observe that for all tuples $(b, c)$, the formula $\phi(x, b, c)$ is not generic in $H_{0}$. However the formula

$$
\psi(x)=\exists y \in P_{1} \exists z \in P_{2} \phi(x, y, z)
$$

whose parameters are from $A$, is realized by $h$ and so it is generic in $H_{0}$. Therefore finite number of translates of $\psi(x)$ cover $H_{0}$, say $H_{0}=\bigcup_{i \leq k} \alpha_{i} \psi(x)$. Take $c$ such that $\operatorname{tp}^{t}(c / A)=\operatorname{tp}^{t}(a / A)$ and $c \downarrow_{A}^{t} \alpha_{1}, \ldots \alpha_{k}$. Thus for a certain element $\alpha \in H_{0}$, we may suppose that $a \in \alpha \psi(x)$ and $a{\underset{A}{\downarrow}}^{t} \alpha$. So $a \in \alpha \phi\left(x, m^{\prime}, \gamma^{\prime}\right)$ for some $m^{\prime} \in k$ and $\gamma^{\prime} \in \Gamma$. By the characterization of the independence (4.3), we have that $a \underset{A, k, \Gamma}{\downarrow} \alpha$ and by transitivity we get $a \underset{A}{\downarrow} \alpha, k, \Gamma$. This is a contradiction since the formula $\alpha \phi\left(x, m^{\prime}, \gamma^{\prime}\right)$ is not generic in $H_{0}$. So we have the claim.

Now since $A$ is $(k, \Gamma)$-independent by lemma (3.2), by transitivity of the independence we see that $h, A \underset{k_{A}, \Gamma_{A}}{\downarrow} k, \Gamma$. As $\operatorname{acl}_{t}\left(k_{A}, \Gamma_{A}\right)=\operatorname{acl}\left(k_{A}, \Gamma_{A}\right)$ by corollary (3.5), it is $(k, \Gamma)$ independent. Therefore, by transitivity and in terms of linear disjointness, we obtain that

$$
\operatorname{acl}(h, A) \underset{k_{A}\left(\Gamma_{A}\right)}{\stackrel{l d}{\downarrow}} k(\Gamma) .
$$

Thus by proposition (3.4), we conclude that $\operatorname{acl}_{t}(h, A)=\operatorname{acl}(h, A)$, and also that $k_{\text {acl }_{t}(h, A)}=k_{A}$ and $\Gamma_{\text {acl }_{t}(h, A)}=\Gamma_{A}$. Since there is a field automorphism over $A$ sending $a$ to $h$, linear disjointness yields a field automorphism over $A \cup k \cup \Gamma$ sending $a$ to $h$. Hence we obtain that $q=p$ and we conclude that $H=H_{0}$.

Remark 5.3. Observe that none of the groups $k^{\times}$and $\Gamma$ satisfy the conclusion of lemma (5.2) as they are not algebraic groups in $\Omega$.

Definition 5.4. (Isogeny) Let $G$ and $H$ be two type-definable groups in a stable theory. We say that $G$ and $H$ are isogenous (or there is an isogeny between them) if there is a type-definable subgroup $S$ of $G \times H$ such that

- The projection of $S$ to $G$, denoted by $G_{S}$, has bounded index (the index is less than the saturation cardinal $\kappa$ ) in $G$, 
- The projection of $S$ to $H$, denoted by $H_{S}$, has bounded index in $H$,

- The kernel $\operatorname{ker}(S)=\{g \in G:(g, 1) \in S\}$ and the co-kernel $\operatorname{coker}(S)=\{h \in H$ : $(1, h) \in S\}$ are finite.

Note that if $G$ and $H$ are isogenous, then there is an isomorphism between $G_{S} / k e r(S)$ and $H_{S} / \operatorname{coker}(S)$. In other words, isogeny is an isomorphism up to a bounded index and finite kernel.

Remark 5.5. Note that the isogeny relation is an equivalence relation. Every group is isogenous to its connected component and every isogeny of the connected component gives rise to an isogeny of the group.

The following lemma is in [2] and it enables us to construct an isogeny between two groups.

Lemma 5.6. [2, 2.4 and 2.5] Let $G_{1}$ and $G_{2}$ be two groups type-definable (type-interpretable) in a stable theory. If there exist parameters $C=\mathrm{acl}^{\mathrm{eq}}(C)$ and elements $a_{1}, b_{1}$ of $G_{1}$ and $a_{2}, b_{2}$ of $G_{2}$ such that

(1) $a_{1}$ and $a_{2}, b_{1}$ and $b_{2}, a_{1} b_{1}$ and $a_{2} b_{2}$ are $C$-interalgebraic

(2) $a_{1}, b_{1}$ and $a_{1} b_{1}$ are pairwise independent over $C$,

then the element $a_{1}$ (respectively $a_{2}$ ) is generic in a unique translate of a connected subgroup $H_{1}$ of $G_{1}$ (respectively $H_{2}$ of $G_{2}$ ), all definable over $C$ and there is an isogeny between $H_{1}$ and $\mathrm{H}_{2}$ given by the stabilizer of the type $\operatorname{tp}\left(a_{1}, a_{2} / C\right)$. In the condition (1), if we just have $a_{2}$ is algebraic over $C, a_{1}$ (respectively for $b_{2}$ and $a_{2} b_{2}$ ), then there is a type-interpretable projection from $H_{1}$ to a quotient of $H_{2}$ by a finite subgroup.

Remark 5.7. The above results can be generalized to the case when both groups $G_{1}$ and $G_{2}$ are $*$-interpretable. In the previous lemma (5.6), suppose that $G_{1}$ is a type-definable connected group and $G_{2}$ is a connected $*$-interpretable group. If the kernel of the projection $N$ is definable, by stability there is a projection with the same kernel from $G_{1}$ to a connected type-interpretable subgroup $D$ of $G_{2}$ whose generic is $C$-interalgebraic with the generic of $G_{2}$. This follows from compactness as $G_{1} / N$ is a type-interpretable group whose generic is a finite tuple and it is $C$-interalgebraic with the generic of $G_{2}$ which is an infinite tuple.

Next we state and prove the Shelah lemma.

Lemma 5.8. (Shelah lemma) Let $T$ be a stable theory. If the type $\operatorname{tp}(A / C)$ is stationary and $B \downarrow_{C} A$, given two $C$-elementary maps $f: A \rightarrow A_{1}$ and $g: B \rightarrow B_{1}$ such that $A_{1} \downarrow_{C} B_{1}$, then $f \cup g$ is also $C$-elementary.

Proof. Since $g$ is $C$-elementary, we have that $g(A) \downarrow_{C} B_{1}$ and $g(A) \equiv_{C} A \equiv_{C} A_{1}$. By stationarity there is an automorphism $h$ fixing $B_{1}$ and sending $g(A)$ to $A_{1}$. Now one can see that $h \circ g$ restricted to $A \cup B$ is $f \cup g$.

Lemma 5.9. Let $v$ and $h$ be generics over a small model $M$ of $T_{t}$ of an algebraic group $V$ defined over $k$ and a type-definable group $H$ in $\Gamma$ respectively. Then we have the independence $v \underset{M}{\downarrow} h$. If $V$ and $H$ are connected, then so is $V \times H$. 
Proof. Suppose that there is a formula $\varphi(x, h)$ over $M$ such that $\models \varphi(v, h)$ and the formula $\varphi(x, h)$ forks over $M$. Let $\phi(x, y)=\varphi(x, y) \wedge\left(x \in P_{1}\right) \wedge\left(y \in P_{2}\right)$. Then by Theorem (2.1), we know that $\phi(x, y)=\bigcup_{i, j} \phi_{i}(x) \wedge \phi_{j}(y)$, where $\phi_{i}(x)$ is a formula defined in $k$ and $\phi_{j}(y)$ is a formula defined in $\Gamma$. Thus for some $i$ or $j$, we obtain that $\phi_{i}(x)$ or $\phi_{j}(y)$ fork. However, a generic over $M$ does not fork over $M$, a contradiction. If $V$ and $H$ are connected, lemma (5.8) yields that the tuple $(v, h)$ is the unique generic of $V \times H$ over $M$ and hence the product is also connected.

Remark 5.10. In stable theories, every type-definable group is an intersection of definable groups in this theory.

The following result was proved by E. Hrushovski and A. Pillay [12]:

Theorem 5.11. [12] Let $A$ be an interpretable group in a one-based stable theory. Then the connected component of $A$ is abelian.

Now we are ready to characterize definable groups in the triple $(\Omega, k, \Gamma)$ in terms of definable and interpretable groups in each sort. We use the group configuration theorem [11] together with lemma (5.6), Theorem (4.3), lemma (4.6), lemma (5.2) and lemma (5.9). Before characterizing definable groups in the triple, we give some examples.

Example 5.12. (Some definable groups in the triple) Algebraic groups over $\Omega$, algebraic groups over $k$, the group $\Gamma$ and its powers, the product $\Omega \times k \times \Gamma$ and

$$
S L(2, k, \Gamma, \Omega)=\left\{\left(\begin{array}{ll}
a & b \\
c & d
\end{array}\right): a d-b c \in k^{\times}\right\} \times\left\{\left(\begin{array}{ll}
a & b \\
c & d
\end{array}\right): a d-b c \in \Gamma\right\}
$$

are all definable in the triple. One can see that each of them satisfy the conclusion of the following Theorem (5.13). The following theorem and its proof are based directly on the result [2, Proposition 2.6].

Theorem 5.13. (Definable Groups) Let $\Omega$ be an algebraically closed field, the field $k$ be a proper subfield of $\Omega$ which is also algebraically closed and $\Gamma$ be a multiplicative subgroup of $\Omega^{\times}$such that $(k, \Gamma)$ is a Mann pair. Any type-definable group in $(\Omega, k, \Gamma)$ is isogenous to a subgroup of an algebraic group. Moreover, any type-definable group $H$ is, up to isogeny, an extension by an algebraic group $V$ of the direct sum of the $k$-rational points of an algebraic group $V_{1}$ over $k$ and a type-interpretable abelian group $H_{1}$ in $\Gamma$ :

$$
0 \longrightarrow V(\Omega) \longrightarrow H \longrightarrow V_{1}(k) \times H_{1}(\Gamma) \longrightarrow 0
$$

Proof. Let $H$ be a type-definable group in $(\Omega, k, \Gamma)$ over some parameters. By remark (5.5), we may suppose that $H$ is connected. We will work over a model containing the parameters defining $H$ which we will omit. Let $T$ be the theory of $\Omega$ in the sense of the field. Given two independent generics $a$ and $b$ of $H$, we write $\bar{a}, \bar{b}$ and $\overline{a \cdot b}$ instead of their algebraic closures in the sense of the triple respectively. Observe that by lemma (4.2) and the characterization of the independence (4.3), the tuple $\overline{a \cdot b}$ is $T$-algebraic over $\bar{a} \cup \bar{b}$ since $\bar{a}, \bar{b}$ are two independent algebraically closed subsets. With the help of the third generic $c$ which is independent from $a$ and $b$, we obtain the following diagram: 


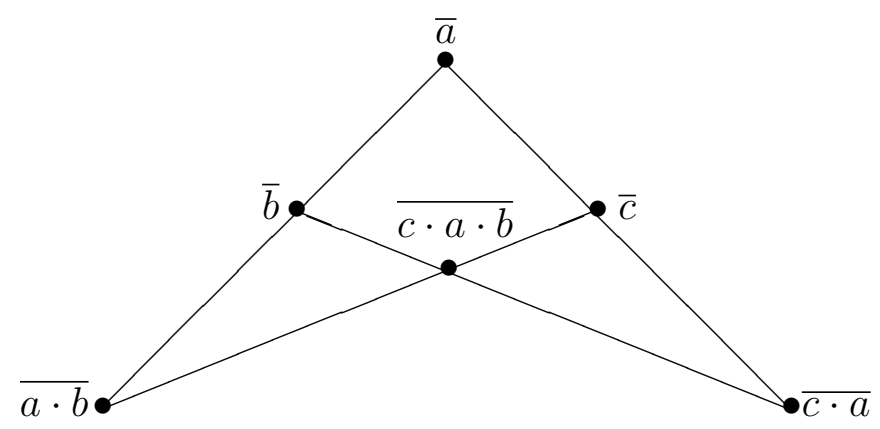

Then by remark (2.4), we have a $T$-group configuration. So by the group configuration theorem [11] and lemma (5.6), there exists a $*$-interpretable group in the pure field $\Omega$ whose generic is interalgebraic with the generic of $H$. As the generic of $H$ is a finite tuple, we conclude that there is an algebraic group in $\Omega$ which $H$ embeds in up to isogeny. Thus up to isogeny, we may assume that $H$ is a subgroup of an algebraic group.

By lemma (4.2) and Theorem (4.3), the set $k_{\overline{a \cdot b}}$ is $k$-algebraic over $k_{\bar{a}} \cup k_{\bar{b}}$. Similarly, the set $\Gamma_{\bar{a} \cdot b}$ is $\Gamma$-algebraic over $\Gamma_{\bar{a}} \cup \Gamma_{\bar{b}}$. Applying the characterization of independence Theorem (4.3) and lemma (4.6), we have the following diagrams:

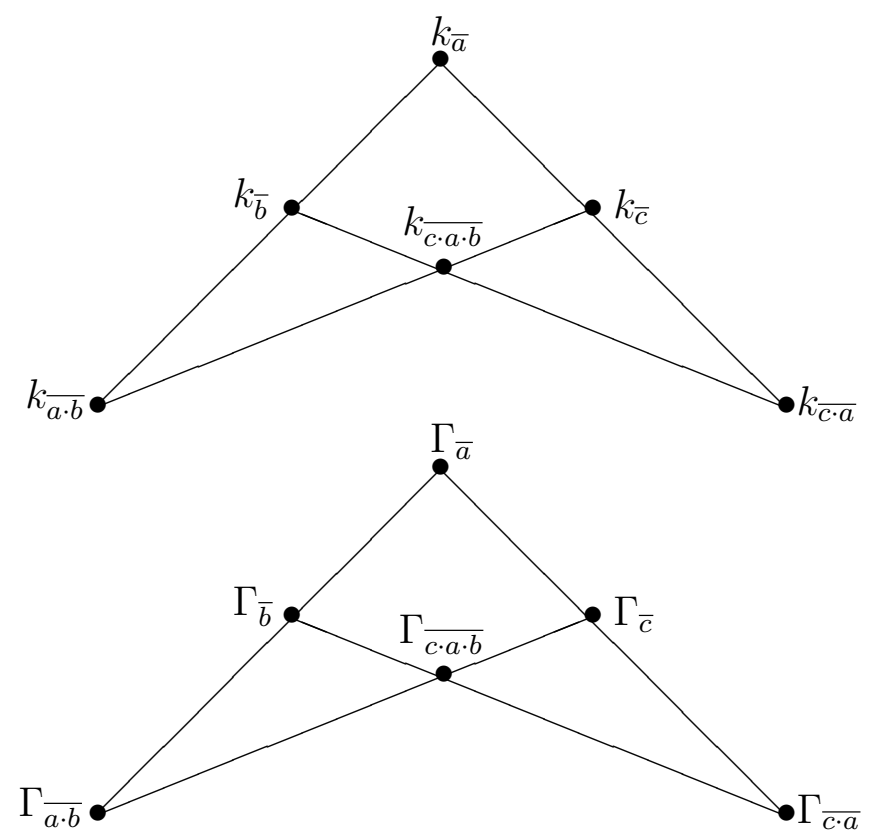

So by the group configuration theorem [11], we obtain a connected $*$-interpretable group $V_{1}$ in $k$ and a connected $*$-interpretable group $H_{1}$ in $\Gamma$. Moreover, a generic $v$ of $V_{1}$ is $k$-interalgebraic with $k_{\bar{a}}$. Similarly, a generic $h$ of $H_{1}$ is $\Gamma$-interalgebraic with $\Gamma_{\bar{a}}$. Now lemma (5.6) yields a projection $\pi_{1}$ from $H$ to a $V_{1}$ and a projection $\pi_{2}$ from $H$ to $H_{1}$. Furthermore the generic $v$ of $V_{1}$ is $T_{t}$-interalgebraic with $k_{\bar{a}}$ and the generic $h$ of $H_{1}$ is $T_{t}$-interalgebraic with $\Gamma_{\bar{a}}$. By lemma (5.9), the tuple $(v, h)$ is a generic of $V_{1} \times H_{1}$ which is $T_{t}$-interalgebraic with $\left(k_{\bar{a}}, \Gamma_{\bar{a}}\right)$. Since $V_{1}$ and $H_{1}$ are connected, the lemma (5.9) also yields that the tuple $(v, h)$ is the only generic of $V_{1} \times H_{1}$ and this product is also connected. Thus by lemma (5.6) again, we have a projection $\pi$ from $H$ to $V_{1} \times H_{1}$ in $k \times \Gamma$ which is given by the stabilizer of the type $\operatorname{tp}^{t}(a, v, h)$. Finally we show that the connected component $N$ of the kernel $k e r(\pi)$ is an algebraic group by lemma (5.2). Let $n$ be a generic of $N$ over $a$ in the sense of the triple. So we have $n \downarrow^{t} a$ and $n \cdot a \bigsqcup^{t} a$. Observe that $n \cdot a \in N a$ is also a generic. As the tuple $(n, 1,1)$ is in the stabilizer of $\operatorname{tp}^{t}(a, v, h)$, 
we have that the tuples $(n \cdot a, v, h)$ and $(a, v, h)$ have the same $t$-type. Thus in particular, we have $k_{\overline{n \cdot a}}=k_{\bar{a}}$ and $\Gamma_{\overline{n \cdot a}}=\Gamma_{\bar{a}}$. Moreover, by lemma (4.2) and Theorem (4.3) we obtain that $k_{\overline{n \cdot a, a}}$ is in the $k$-algebraic closure of $k_{\overline{n \cdot a}}$ and $k_{\bar{a}}$. Therefore we see that $k_{\overline{n \cdot a, a}}=k_{\bar{a}}$ and similarly $\Gamma_{\bar{n} \cdot a, a}=\Gamma_{\bar{a}}$. Now the type $\operatorname{tp}^{t}(n \cdot a / \bar{a})$ satisfies the hypothesis of lemma (5.2) and we conclude that $N$ is an algebraic group. By elimination of imaginaries in $k$ and by remark (5.7), we can take $V_{1}$ to be an algebraic group and $H_{1}$ to be type-interpretable. Note also that $H_{1}$ is abelian by (5.11) and remark (5.10), as pure abelian groups are one-based.

\section{IMAGINARIES AND INTERPRETABLE Groups}

Our goal in this section is to characterize interpretable groups in the triple $(\Omega, k, \Gamma)$. Throughout the section, we assume that $\Gamma$ is divisible. As $(k, \Gamma)$ is a Mann pair, taking $n=1$ in the equation (1), we see that $k \cap \Gamma$ is finite, thus the intersection is a finite subset of the group of roots of unity in $\Omega$. In this case, the group $\Gamma$ is torsion-free and hence it is strongly minimal by [16, Corollary 3.1.11]. For every algebraically closed subset $A$ in the triple, its $\Gamma$-part $\Gamma_{A}$ contains $\Gamma_{0}$ and so $\Gamma_{A}$ is an elementary substructure of $\Gamma$. By [5, Proposition 8.3], the triple is $\omega$-stable with infinite Morley rank. In this section, we will give a description of imaginaries in the triple in terms of real elements and the motivation comes from [17]. This enables us to characterize interpretable groups in $(\Omega, k, \Gamma)$. Observe that $(\Omega, k, \Gamma)$ does not eliminate imaginaries. Our description of imaginaries will be by means of canonical bases as studied in [17].

Lemma 6.1. Let $B$ be an elementary substructure of $(\Omega, k, \Gamma)$. Suppose that

$$
d=\operatorname{Cb}(\operatorname{tp}(a / \operatorname{acl}(B, k, \Gamma))) .
$$

Then $a \stackrel{t}{\downarrow} B, k, \Gamma$.

Proof. As $\Omega$ eliminates imaginaries as a pure field, we might assume that $d$ is contained in $\Omega$. First of all, note that $\operatorname{acl}_{t}(B, k, \Gamma)=\operatorname{acl}(B, k, \Gamma)$ and $a \underset{d}{\downarrow} B, k, \Gamma$ as $d$ is the canonical base. The independence $a \underset{d}{\downarrow} B, k, \Gamma$ yields that

$$
\operatorname{acl}(a, d) \underset{d, k_{\mathrm{acl}_{t}(d)}, \Gamma_{\mathrm{acl}_{t}(d)}}{\downarrow} k, \Gamma .
$$

Note that by corollary (3.5), we see that

$$
\operatorname{acl}_{t}(d)=\operatorname{acl}\left(d, k_{\operatorname{acl}_{t}(d)}, \Gamma_{\operatorname{acl}_{t}(d)}\right)
$$

and

$$
\operatorname{acl}_{t}\left(k_{\operatorname{acl}_{t}(d)}, \Gamma_{\operatorname{acl}_{t}(d)}\right)=\operatorname{acl}\left(k_{\operatorname{acl}_{t}(d)}, \Gamma_{\operatorname{acl}_{t}(d)}\right) .
$$

In particular, they are $(k, \Gamma)$-independent by lemma (3.2). Since

$$
d \underset{\text { acl }_{t}(d), \Gamma_{\mathrm{acl}_{t}(d)}}{\downarrow} k, \Gamma
$$


by lemma (3.2) again, from (18), (19) and transitivity we obtain that

$$
\operatorname{acl}\left(a, d, k_{\text {acl }_{t}(d)}, \Gamma_{\operatorname{acl}_{t}(d)}\right) \underset{k_{\operatorname{acl}_{t}(d)}, \Gamma_{\operatorname{acl}_{t}(d)}}{\downarrow} k, \Gamma .
$$

Combining (20), lemma (3.2) and transitivity, in terms of linear disjointness we see that

$$
\operatorname{acl}\left(a, d, k_{\mathrm{acl}_{t}(d)}, \Gamma_{\mathrm{acl}_{t}(d)}\right) \underbrace{l d}_{k_{\mathrm{acl}_{t}(d)}\left(\Gamma_{\mathrm{acl}_{t}(d)}\right)} k(\Gamma)
$$

as the $k$-part of $\operatorname{acl}\left(k_{\operatorname{acl}_{t}(d)}, \Gamma_{\operatorname{acl}_{t}(d)}\right)$ is $k_{\operatorname{acl}_{t}(d)}$ and the $\Gamma$-part of $\operatorname{acl}\left(k_{\mathrm{acl}_{t}(d)}, \Gamma_{\operatorname{acl}_{t}(d)}\right)$ is $\Gamma_{\operatorname{acl}_{t}(d)}$. Thus by proposition (3.4), we deduce that

$$
\operatorname{acl}_{t}\left(a, d, k_{\operatorname{acl}_{t}(d)}, \Gamma_{\operatorname{acl}_{t}(d)}\right)=\operatorname{acl}\left(a, d, k_{\operatorname{acl}_{t}(d)}, \Gamma_{\operatorname{acl}_{t}(d)}\right),
$$

and also that $k_{\operatorname{acl}_{t}(a, d)}=k_{\operatorname{acl}_{t}(d)}$ and $\Gamma_{\operatorname{acl}_{t}(a, d)}=\Gamma_{\operatorname{acl}_{t}(d)}$. Now as $a \underset{d}{\downarrow} B, k, \Gamma$, we have that

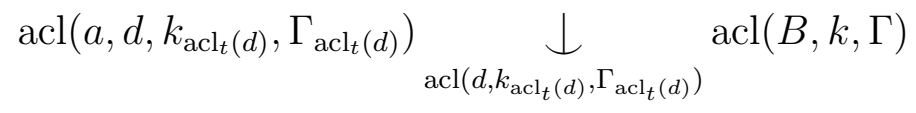

and this yields by corollary (3.5) that

$$
\operatorname{acl}_{t}(a, d) \underset{\operatorname{acl}_{t}(d)}{\perp} \operatorname{acl}_{t}(B, k, \Gamma) .
$$

We finish the proof by the characterization of the independence Theorem (4.3).

The idea of the next corollary is due to [1, Proposition 7.5].

Corollary 6.2. Let $B$ be an elementary substructure of $(\Omega, k, \Gamma)$ and let a be a finite tuple from $\Omega$. Put $d=\operatorname{Cb}(\operatorname{tp}(a / \operatorname{acl}(B, k, \Gamma)))$. Then $\operatorname{Cb}\left(\operatorname{tp}^{t}(a / B)\right)$ is interalgebraic in $(\Omega, k, \Gamma)$ with $\mathrm{Cb}\left(\operatorname{tp}^{t}(d / B)\right)$.

Proof. Set $p=\operatorname{tp}^{t}(a / B)$ and $q=\operatorname{tp}^{t}(d / B)$. Let $e_{1}=\mathrm{Cb}(p)$ and $e_{2}=\operatorname{Cb}(q)$. Note that $e_{1}$ and

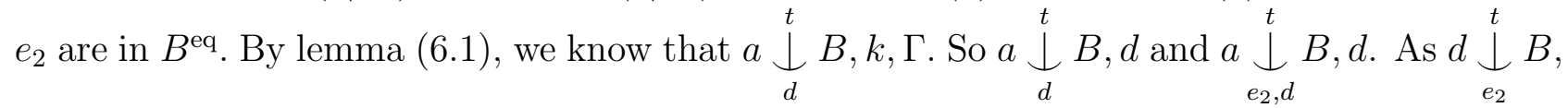
by transitivity we conclude that $a \stackrel{t}{\downarrow} B$. This gives that $e_{1}$ is algebraic over $e_{2}$ in the sense of the triple.

Now we show that $e_{2}$ is algebraic over $e_{1}$ in the sense of the triple. Take $B_{1}$ such that $\operatorname{tp}^{t}\left(B_{1} / e_{1}\right)=\operatorname{tp}^{t}\left(B / e_{1}\right)$ and $B_{1} \underset{e_{1}}{\downarrow} B$. Let $p_{B_{1}}$ and $q_{B_{1}}$ be the corresponding types. Choose an element $a_{1} \models p_{B} \cup p_{B_{1}}$ such that $a_{1} \underset{e_{1}}{\stackrel{t}{\perp}} B, B_{1}$. Put $d_{1}=\operatorname{Cb}\left(\operatorname{tp}\left(a_{1} / \operatorname{acl}\left(B, B_{1}, k, \Gamma\right)\right)\right)$, the element $d_{2}=\mathrm{Cb}\left(\operatorname{tp}\left(a_{1} / \operatorname{acl}(B, k, \Gamma)\right)\right)$ and $d_{3}=\operatorname{Cb}\left(\operatorname{tp}\left(a_{1} / \operatorname{acl}\left(B_{1}, k, \Gamma\right)\right)\right)$. The independence $a_{1}{\underset{e}{e_{1}}}^{t} B, B_{1}$ yields that $a_{1} \underset{B}{\downarrow} B_{1}$ and by the characterization of the independence Theorem (4.3), we obtain that

$$
a_{1} \underset{B, k, \Gamma}{\downarrow} B_{1}
$$


Similarly we have

$$
a_{1} \underset{B_{1}, k, \Gamma}{\downarrow} B
$$

Thus by $(21)$ and $(22)$, the types $\operatorname{tp}\left(a_{1} / \operatorname{acl}(B, k, \Gamma)\right)$ and $\operatorname{tp}\left(a_{1} / \operatorname{acl}\left(B_{1}, k, \Gamma\right)\right)$ have a common non-forking extension $\operatorname{tp}\left(a_{1} / \operatorname{acl}\left(B, B_{1}, k, \Gamma\right)\right)$, in other words they are parallel types. Hence we conclude that $d_{1}=d_{2}=d_{3}$, as a canonical base is the minimal base over which the type does not fork. By the choice of the element $a_{1}$, we have that $d_{1} \models q_{B} \cup q_{B_{1}}$. Note that any triple automorphism fixes $k$ and $\Gamma$ setwise. Thus any automorphism which fixes $a_{1}$ and $B$ also fixes the type $\operatorname{tp}\left(a_{1} / \operatorname{acl}(B, k, \Gamma)\right)$ setwise. This yields that the element $d_{1}$ is in $\operatorname{acl}_{t}\left(a_{1}, B\right) \cap \operatorname{acl}_{t}\left(a_{1}, B_{1}\right)$ as it is the canonical base of the types $\operatorname{tp}\left(a_{1} / \operatorname{acl}(B, k, \Gamma)\right)$ and $\operatorname{tp}\left(a_{1} / \operatorname{acl}\left(B_{1}, k, \Gamma\right)\right)$. Now, from $a_{1} \underset{B_{1}}{\downarrow} B$ and $d_{1} \in \operatorname{acl}_{t}\left(a_{1}, B_{1}\right)$, we obtain that $d_{1} \underset{B_{1}}{\downarrow} B$. By the

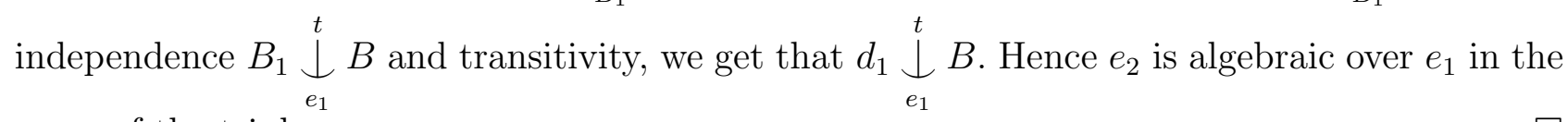
sense of the triple.

Definition 6.3. Let $T$ be a stable theory. Let $a$ be a tuple and $A$ be set of parameters (possibly containing imaginary elements), and $D$ be a definable subset. We say that the type $\operatorname{tp}(a / A)$ is almost $D$-internal if there exists a set of parameters $B$ such that $a \underset{A}{\downarrow} B$ and $a \in \operatorname{acl}(A, B, D)$.

We say that a type in the triple $(\Omega, k, \Gamma)$ is almost $(k, \Gamma)$-internal if it is almost $k \cup \Gamma$-internal. The next lemma asserts that, up to interalgebraicity, an imaginary element in the triple is a canonical base of a type over itself and this type is almost $(k, \Gamma)$-internal. The proof of the following lemma is based on [17, Lemma 2.2].

Lemma 6.4. Let $e \in(\Omega, k, \Gamma)^{\mathrm{eq}}$ be an imaginary element. There is $e^{\prime} \in(\Omega, k, \Gamma)^{\mathrm{eq}}$ interalgebraic with $e$, such that for some finite tuple $d^{\prime}$ from $\Omega$ we have $e^{\prime}=\operatorname{Cb}\left(\operatorname{tp}^{t}\left(d^{\prime} / e^{\prime}\right)\right)$ and $\operatorname{tp}^{t}\left(d^{\prime} / e^{\prime}\right)$ is almost $(k, \Gamma)$-internal.

Proof. Let $a$ be a tuple in $\Omega$ such that $e=f(a)$ for some $\emptyset$-definable function in $(\Omega, k, \Gamma)^{\text {eq }}$. Set $e_{1}=\operatorname{Cb}\left(\operatorname{tp}^{t}\left(a / \operatorname{acl}_{t}^{e q}(e)\right)\right)$. Observe that $e_{1}$ is algebraic over $e$. As $e=f(a)$ and $a \perp_{e_{1}}^{t} e$, we obtain that $e \underset{e_{1}}{\downarrow} e$ and hence $e$ and $e_{1}$ are interalgebraic. Now let $B$ be an elementary substructure of $(\Omega, k, \Gamma)$ such that $e_{1} \in B^{\text {eq }}$ and $a \stackrel{t}{\downarrow} B$. Let $d^{\prime}=\operatorname{Cb}(\operatorname{tp}(a / \operatorname{acl}(B, k, \Gamma)))$. We may assume $d^{\prime}$ to be a finite tuple in $\Omega$ due to the $\omega$-stability and elimination of imaginaries. Put

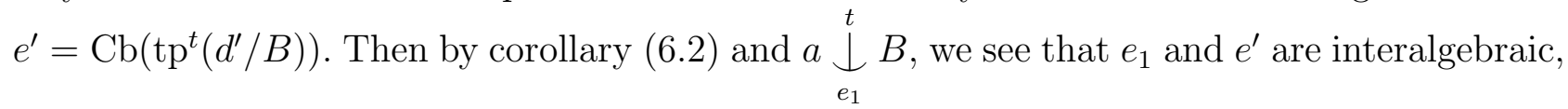
and hence $e$ and $e^{\prime}$ are interalgebraic. Note that the type $\operatorname{tp}^{t}\left(d^{\prime} / B\right)$ is almost $(k, \Gamma)$-internal. Thus, the type $\operatorname{tp}^{t}\left(d^{\prime} / e^{\prime}\right)$ is also almost $(k, \Gamma)$-internal since $d^{\prime} \underset{e^{\prime}}{\stackrel{t}{\perp} B}$.

The next lemma is motivated from [17, Lemma 1.8].

Lemma 6.5. Let $e \in(\Omega, k, \Gamma)^{\mathrm{eq}}$ and $B=\operatorname{acl}_{t}(e) \cap(k \cup \Gamma)$. Let $c$ be a tuple from $k \cup \Gamma$. Then $\operatorname{tp}^{t}(c / B, e)$ is finitely satisfiable in $B$. 
Proof. By corollary (4.5), we see that

$$
\operatorname{acl}_{t}(e){\underset{B}{t}}_{B}^{t} k, \Gamma .
$$

Note that $k_{\text {acl }_{t}(e)}$ is an elementary substructure of $k$ and $\Gamma_{\operatorname{acl}_{t}(e)}$ is an elementary substructure of $\Gamma$. By corollary (3.8), we see that $\operatorname{acl}(B)=\operatorname{acl}\left(k_{\operatorname{acl}_{t}(e)}, \Gamma_{\operatorname{acl}_{t}(e)}\right)$ is an elementary substructure of $\Omega$ in the sense of the triple and

$$
\operatorname{acl}(B) \cap(k \cup \Gamma)=k_{\operatorname{acl}_{t}(e)} \cup \Gamma_{\operatorname{acl}_{t}(e)}=B .
$$

Now by equation (23) and [21, Corollary 8.3.7], we have that the type $\operatorname{tp}^{t}(c / \operatorname{acl}(B), e)$ is finitely satisfiable in $\operatorname{acl}(B)$. On the other hand, if $c$ is in $k \cup \Gamma$ and $\operatorname{acl}(B)$, then it is in $B$ by (24). Hence we can say that any $c \in \operatorname{acl}(B)$ which satisfies a finite part of $\operatorname{tp}^{t}(c / B, e)$ has to be in $B$ as well.

Now we recall a theorem which ensures the definability of Morley rank.

Theorem 6.6. [22, Chapter 4, 4.7.10] Let $T$ be an w-stable theory. Suppose that there exist strongly minimal formulas $\phi_{1}, \ldots, \phi_{k}$ such that any type is non-orthogonal to a certain $\phi_{i}$. Then $T$ has finite Morley rank and Morley rank is definable, that is to say for every formula $\theta(x, y)$ and every natural number $n<\omega$ the set $\{a: \operatorname{MR}(\theta(x, a))=n\}$ is definable.

For the following definition, we follow [4]. Given sets $X$ and $Y$, we write

$$
f: X \stackrel{\mathrm{n}}{\longrightarrow} Y
$$

to indicate that $f$ is a map from $X$ to the power set of $Y$ such that $|f(x)| \leq n$ for all $x \in X$. For such a map $f$, its graph $G r a p h(f)$ is the set

$$
\{(x, y) \in X \times Y: y \in f(x)\},
$$

and for a subset $Z$ of $X$, we set $f(Z)=\bigcup_{x \in Z} f(x)$. If $X$ and $Y$ are definable in a structure, then such an $f$ is called definable if $\operatorname{Graph}(f)$ is, and for short we say that $f$ is $n$-definable. Clearly, 1- definable functions are ordinary definable functions.

The following lemma and its proof are directly based on [17, 2.4 and 2.5].

Lemma 6.7. Let $e \in(\Omega, k, \Gamma)^{\mathrm{eq}}$ be an imaginary element. There is a tuple $d$ from $\Omega$, an $L_{t}$-definable function $f(x)$ over $\emptyset$, an $L_{t}$-formula $\psi(y)$ over $e$ and an $L_{t}$-n-definable function $h\left(y, z_{1}, z_{2}\right)$ over e for some $n$ such that

(i) $f(d)=e$,

(ii) $\psi(y) \in \operatorname{tp}(d / e)$,

(iii) $\left(\forall y, y^{\prime}\right)\left(\psi(y) \wedge \psi\left(y^{\prime}\right) \Longrightarrow \exists z_{1} \exists z_{2}\left(P_{1}\left(z_{1}\right) \wedge P_{2}\left(z_{2}\right) \wedge y^{\prime} \in h\left(y, z_{1}, z_{2}\right)\right)\right)$

(iv) Moreover $d$ is $T_{t}$-independent from $k \cup \Gamma$ over $e$.

Proof. For $(i),(i i)$ and $(i i i)$ we refer the reader to [17, 2.4], as the verbatim proof of [18, Lemma 7.4.2] (see also [23, Proposition 3.4.9]) yields the function $h$ if we replace dcl by acl. Without loss of generality, we may assume that $h$ is an ordinary definable function. Now we prove $(i v)$. Let $(f, \psi, h)$ be fixed as in the lemma. Choose $d$ such that $\operatorname{MR}\left(\operatorname{tp}^{t}(d / e)\right)$ is minimized and also 
(i)-(iii) are satisfied with respect to $(f, \psi, h)$. By lemma (6.4) we can assume that the type $q=\operatorname{tp}^{t}(d / e)$ is almost $(k, \Gamma)$-internal. We will show that $d$ is independent from $k, \Gamma$ over $e$. Suppose not and choose $b=\left(b_{1}, b_{2}\right) \in k \times \Gamma$ such that $d$ forks with $b$ over $e$. Note that by almost internality and as $k$ and $\Gamma$ are strongly minimal, we deduce that $\operatorname{MR}\left(\operatorname{tp}^{t}(d / e)\right)$ is finite. Let $m=\operatorname{MR}\left(\operatorname{tp}^{t}(d / e, b)\right)<\operatorname{MR}\left(\operatorname{tp}^{t}(d / e)\right)$. By almost internality again, there is a formula $\varphi(x)$ in $q$ whose all realisations are almost $(k, \Gamma)$-internal. Now consider the induced structure on $\varphi \cup k \cup \Gamma$ with two strongly minimal formulas $k$ and $\Gamma$. We apply Theorem (6.6) to this induced structure. Thus by Theorem (6.6), there is a formula $\chi\left(y, z_{1}, z_{2}\right)$ in $q$ such that $\chi\left(d, b_{1}, b_{2}\right)$ holds, $\operatorname{MR}\left(\chi\left(y, b_{1}, b_{2}\right)\right)=m$ and a formula $\theta\left(z_{1}, z_{2}\right)$ satisfied by $\left(b_{1}, b_{2}\right)$ such that for any $c=\left(c_{1}, c_{2}\right)$ we have $\operatorname{MR}\left(\chi\left(y, c_{1}, c_{2}\right)\right)=m$ if and only if $\theta\left(c_{1}, c_{2}\right)$ holds. In other words, we can say that $\operatorname{MR}\left(\chi\left(y, c_{1}, c_{2}\right)\right)=m$ if and only if $\chi\left(y, c_{1}, c_{2}\right)$ is consistent. Let $\Delta\left(z_{1}, z_{2}\right)$ be the formula

$$
\begin{gathered}
\exists y(f(y)=e \wedge \psi(y)) \wedge\left(\forall y_{1}, y_{2}\right)\left(\psi\left(y_{1}\right) \wedge \psi\left(y_{2}\right)\right) \Longrightarrow \\
\left(h\left(y_{1}, z_{1}, z_{2}\right)=y_{2} \wedge P_{1}\left(z_{1}\right) \wedge P_{2}\left(z_{2}\right)\right) .
\end{gathered}
$$

Observe that $\Delta\left(b_{1}, b_{2}\right)$ holds. Let $B=\operatorname{acl}_{t}(e) \cap(k \cup \Gamma)$. By lemma $(6.5)$, there is $r=\left(r_{1}, r_{2}\right) \in B$ such that $\Delta\left(r_{1}, r_{2}\right)$ holds. Then we find $d_{1}$ satisfying $(i),(i i)$ and $(i i i)$ of the lemma with $\chi\left(d_{1}, r_{1}, r_{2}\right)$ holds. Since $r$ is algebraic over $e$, we have that $\operatorname{MR}\left(\operatorname{tp}^{t}\left(d_{1} / e\right)\right) \leq m$, contradicting the choice of $d$.

Combining lemmas (6.4) and (6.7), we obtain the following theorem which is a description of imaginaries in terms of real elements:

Theorem 6.8. Let $e \in(\Omega, k, \Gamma)^{\mathrm{eq}}$ be an imaginary element. There is a finite real tuple $d$ such that $e$ is algebraic over $d$, the type $\operatorname{tp}^{t}(d / e)$ is almost $(k, \Gamma)$-internal and $d$ is independent from $k \cup \Gamma$ over $e$ in the sense of the triple.

Next, we characterize interpretable groups in the triple. We start with a lemma from [2]:

Lemma 6.9. [2, Lemma 3.1] Let $H$ be a connected interpretable group in a stable theory. Let $\alpha, \beta$ and $\gamma$ be three independent generics of $H$ and $a_{0}$ be a real element such that $\alpha$ is algebraic over $a_{0}$. Then there exist real tuples $a, b, c, d$, e and $f$ such that

$$
(a, \alpha) \equiv(b, \beta) \equiv(c, \gamma) \equiv(d, \alpha \beta) \equiv(e, \gamma \alpha) \equiv(f, \gamma \alpha \beta) \equiv\left(a_{0}, \alpha\right)
$$

and

$$
a \underset{\alpha}{\downarrow} b, c, d, e, f
$$

and the same for the other tuples. Moreover in the following diagram

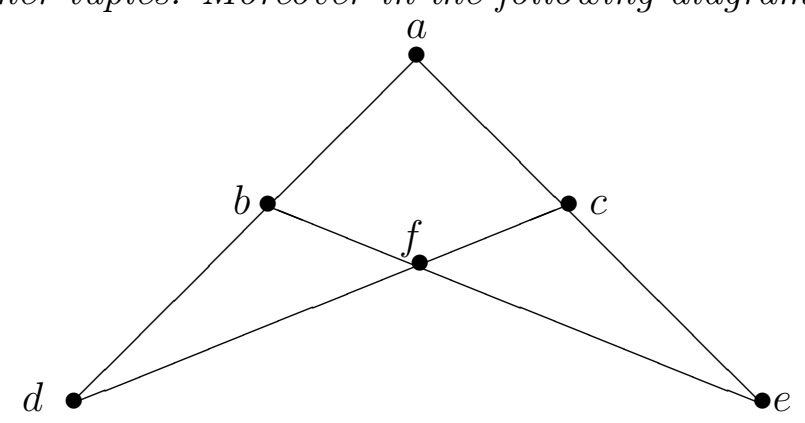

all non-linear triples are independent and each point is independent from each line which does not contain it. 
We need two more lemmas for interpretable groups.

Lemma 6.10. If $\operatorname{tp}^{t}(a / A)$ is almost $(k, \Gamma)$-internal over a real set of parameters $A$, then $a \in$ $\operatorname{acl}(A, k, \Gamma)$.

Proof. Take $B=\operatorname{acl}_{t}(B)$ containing $A$ such that $a \stackrel{t}{\downarrow}_{A}^{t} B$ and $a \in \operatorname{acl}_{t}(B, k, \Gamma)=\operatorname{acl}(B, k, \Gamma)$. The characterization of the independence (4.3) yields that $a \underset{\downarrow}{\downarrow} B$ and therefore we obtain that $a \in \operatorname{acl}(A, k, \Gamma)$.

Lemma 6.11. Let $H$ be a definable group in the sense of the triple. If a generic of $H$ is almost $(k, \Gamma)$-internal then $H$ is isogenous to a cartesian product of $k$-rational points of an algebraic group defined over $k$ and an interpretable group in $\Gamma$.

Proof. By almost internality, we deduce that $H$ is of finite Morley rank. By [4, 6.4], the pair $(\Omega, \Gamma)$ is $\omega$-stable and $\operatorname{MR}(\Omega, \Gamma)=\omega$. Thus infinite algebraic groups have infinite Morley rank in the triple as

$$
\operatorname{MR}(\Omega, k, \Gamma) \geq \omega .
$$

We conclude by Theorem (5.13) and $\omega$-stability.

Now we are ready to characterize interpretable groups in the triple and it demands all the tools we have proved so far. The following theorem and its proof are directly based on $[2$, Theorem 3.5].

Theorem 6.12. (Interpretable groups) Let $\Omega$ be an algebraically closed field, the field $k$ be a proper subfield of $\Omega$ which is also algebraically closed and $\Gamma$ be a divisible multiplicative subgroup of $\Omega^{\times}$such that $(k, \Gamma)$ is a Mann pair. Every interpretable group $H$ in $(\Omega, k, \Gamma)$ is, up to isogeny, an extension of a direct sum of $k$-rational points of an algebraic group $V_{1}$ over $k$ and an interpretable abelian group $H_{1}$ in $\Gamma$ by an interpretable group $N$, which is a quotient of an algebraic group $W$ by a subgroup $N_{1}$ which in turn is isogenous to a cartesian product of $k$-rational points of an algebraic group $V_{2}$ over $k$ and an interpretable abelian group $\mathrm{H}_{2}$ in $\Gamma$ :

$$
0 \longrightarrow N \longrightarrow H \longrightarrow V_{1}(k) \times H_{1}(\Gamma) \longrightarrow 0
$$

with

$$
0 \longrightarrow V_{2}(k) \times H_{2}(\Gamma) \longrightarrow W \longrightarrow N \longrightarrow 0
$$

Proof. Let $H$ be an interpretable group in $(\Omega, k, \Gamma)$. By remark (5.5), we may suppose that $H$ is connected. Again we work over a small model that we omit. Let $\alpha, \beta$ and $\gamma$ be three independent generics of $H$ in the sense of the triple. By Theorem (6.8), the generic $\alpha$ is algebraic over a real tuple $a_{0}$ which is $T_{t}$-independent from $k, \Gamma$ over $\alpha$ and the type $\operatorname{tp}^{t}\left(a_{0} / \alpha\right)$ is almost $(k, \Gamma)$-internal. Then by lemma (6.9), there are real tuples $a, b, c, d, e$ and $f$ such that

$$
(a, \alpha) \equiv^{t}(b, \beta) \equiv^{t}(c, \gamma) \equiv^{t}(d, \alpha \cdot \beta) \equiv^{t}(e, \gamma \cdot \alpha) \equiv^{t}(f, \gamma \cdot \alpha \cdot \beta) \equiv^{t}\left(a_{0}, \alpha\right)
$$

and if we put $\bar{a}=\operatorname{acl}_{t}(a)$ and the same for the others, we have the following diagram: 


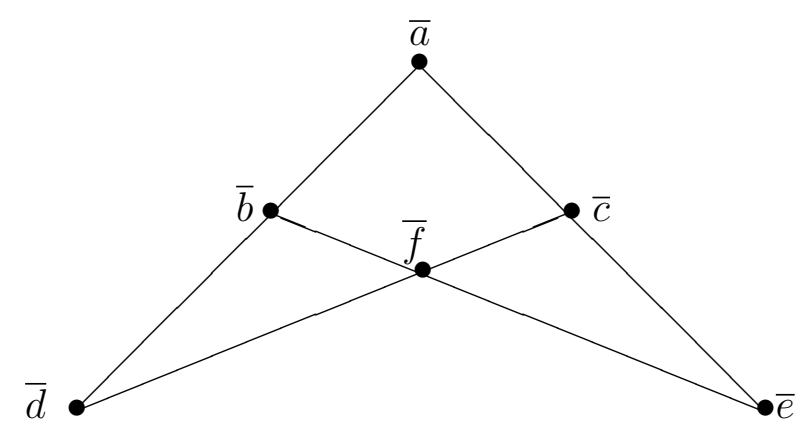

such that each non-colinear triples of them are $T_{t}$-independent and each set is $T_{t}$-independent from the lines which do not contain it. Since $a \stackrel{t}{\downarrow} k, \Gamma$, we see that $k_{\bar{a}} \subset \operatorname{acl}_{t}(\alpha) \subset \bar{a}$. Therefore, we obtain that $k_{\bar{a}}=\operatorname{acl}_{t}(\alpha) \cap k$. Moreover, by lemma (4.2) and Theorem (4.3), we have that $k_{\text {acl }_{t}(\bar{a}, \bar{b})}=\operatorname{acl}\left(k_{\bar{a}}, k_{\bar{b}}\right)$. Since $k_{\bar{d}}=\operatorname{acl}_{t}(\alpha \cdot \beta) \cap k \subset k_{\operatorname{acl}_{t}(\bar{a}, \bar{b})}$, we get that $k_{\bar{d}} \subset \operatorname{acl}\left(k_{\bar{a}}, k_{\bar{b}}\right)$. This is true for all other tuples and by Theorem (4.3), the set $k_{\bar{a}}$ is independent in the field sense from $\operatorname{acl}\left(k_{\bar{b}}, k_{\bar{e}}\right)$ and the same for the others. We have the same thing for the group $\Gamma$ by lemma (4.6). So we have the following diagrams:

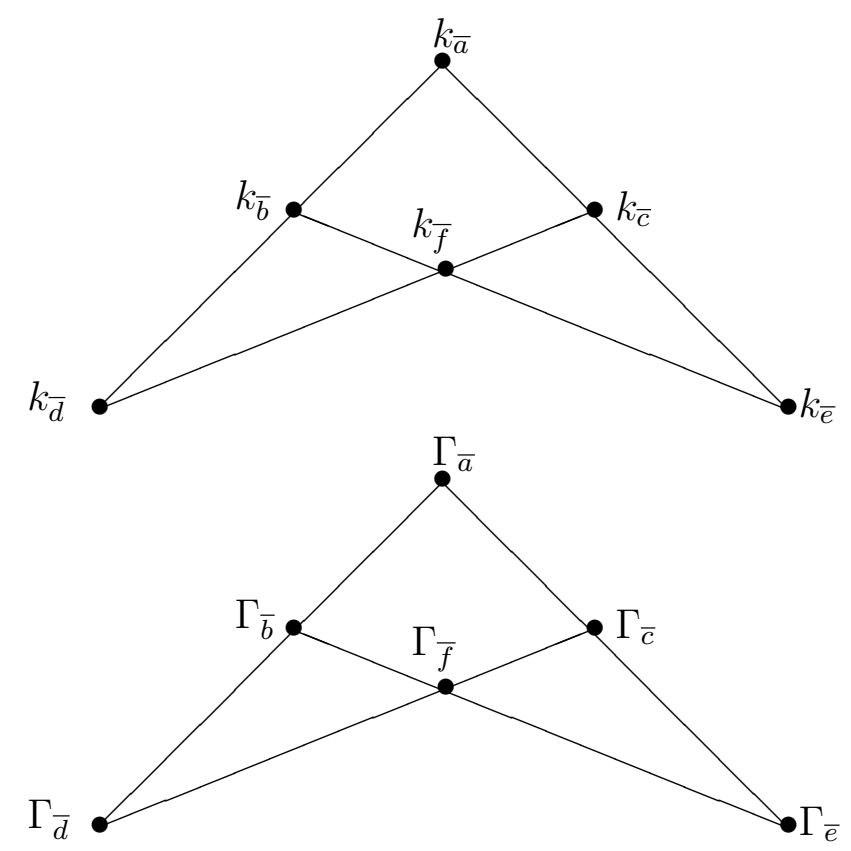

and by the group configuration theorem [11], there is a connected $*$-interpretable group $V_{1}(k)$ in $k$ whose generic $v$ is $k$-interalgebraic with $k_{\bar{a}}$. By $\omega$-stability, lemma (5.6) and since $k$ eliminates imaginaries, we may assume that $V_{1}$ is an algebraic group and its generic $v$ is interalgebraic with $k_{\bar{a}}$ in the sense of the triple. Similarly, there exists a connected interpretable group $H_{1}(\Gamma)$ in $\Gamma$ whose generic $h$ is interalgebraic with $\Gamma_{\bar{a}}$ in $T_{t}$. Furthermore, by (5.11), the group $H_{1}$ is abelian. By lemmas $(5.9)$, the tuple $(v, h)$ is the generic of $V_{1} \times H_{1}$ which is $T_{t}$-interalgebraic with $\left(k_{\bar{a}}, \Gamma_{\bar{a}}\right)$. Moreover, by lemma (5.6), we have a projection $\pi$ from $H$ to the connected group $V_{1} \times H_{1}$ in $k \times \Gamma$ which is given by the stabilizer of the type $\operatorname{tp}^{t}(a, v, h)$.

Next we show that the points $\bar{a}, \bar{b}, \bar{c}, \bar{d}, \bar{e}, \bar{f}$ give a $T$-group configuration with the help of the parameter set $k, \Gamma$. We know that all three non-colinear of them are independent in the sense of the triple. As $\beta$ is algebraic over $b$ and $\alpha \beta$ is algebraic over $d$, we have that $\alpha$ is algebraic over $b, d$. Moreover by lemma $(4.2)$, we know that $\operatorname{acl}(\bar{b}, \bar{d})=\operatorname{acl}_{t}(\bar{b}, \bar{d})$. Since the type $\operatorname{tp}^{t}(a / \alpha)$ 
is almost $(k, \Gamma)$-internal and $\alpha$ is algebraic over $b, d$, we observe that the type $\operatorname{tp}^{t}(a / \operatorname{acl}(\bar{b}, \bar{d}))$ is also almost $(k, \Gamma)$-internal. Thus by lemma $(6.10)$ we obtain that $a \in \operatorname{acl}(k, \Gamma, \bar{b}, \bar{d})$. The same holds for the other tuples. Therefore, we obtain a connected $*$-interpretable group $V$ over $\operatorname{acl}(k, \Gamma)$ in the field sense and two independent generics $a_{1}, b_{1}$ of $V$ such that $a_{1}$ is field interalgebraic with $\bar{a}$ over $k, \Gamma$, the element $b_{1}$ is field interalgebraic with $\bar{b}$ and $a_{1} \cdot b_{1}$ is field interalgebraic with $\bar{d}$. Since the tuples $\alpha, \beta$ and $\gamma$ (they are finite tuples) are algebraic over some finite subtuples of $a_{1}, b_{1}$ and $a_{1} \cdot b_{1}$ respectively and as $V$ is a connected pro-algebraic group, there exists a connected algebraic group $W$ over $\operatorname{acl}(k, \Gamma)$ and two independent generics $a_{2}, b_{2}$ such that $\alpha$ is algebraic over $a_{2}$ and the same for the others. Note that $a_{2}$ is field algebraic over $k, \Gamma, \bar{a}$ and the same for the others. Moreover, since $\bar{a}, \bar{b}$ and $\bar{d}$ are pairwise $T_{t}$-independent over $k, \Gamma$, then so are $a_{2}, b_{2}$ and $a_{2} \cdot b_{2}$. As $\alpha$ is algebraic over $a$ and $a \underset{k_{\bar{a}}, \Gamma_{\bar{a}}}{\downarrow} k, \Gamma$ by corollary (4.5), we see that $\alpha \stackrel{t}{\perp} k, \Gamma$. Now let $N$ be the connected component of $\operatorname{ker}(\pi)$. Then $\alpha$ is generic in $k_{\bar{a}}, \Gamma_{\bar{a}}$

$N \alpha$ over $\operatorname{acl}_{t}(v, h)=\operatorname{acl}_{t}\left(k_{\bar{a}}, \Gamma_{\bar{a}}\right)$, so $\alpha$ is also generic over $k \cup \Gamma$.

Now we apply the lemma (5.6) to the tuples $\left(a_{2}, \alpha\right)$ and $\left(b_{2}, \beta\right)$. So this gives us a surjection $\phi$ from $W$ to $N$, up to isogeny. Lastly, we show that the connected component $N_{1}$ of $k e r(\phi)$ is isogenous to a cartesian product of $k$-rational points of an algebraic group defined over $k$ and an interpretable group in $\Gamma$. Let $n_{1}$ be a generic of $N_{1}$ over $k, \Gamma$ and $a_{2}$. Then the point $\left(n_{1}, 1_{N}\right)$ is in the stabilizer of the type $\operatorname{tp}^{t}\left(a_{2}, \alpha / \operatorname{acl}^{\mathrm{eq}}(k, \Gamma)\right)$ and so $\operatorname{tp}^{t}\left(n_{1} \cdot a_{2} / \alpha\right)=\operatorname{tp}^{t}\left(a_{2} / \alpha\right)$. Since $\operatorname{tp}^{t}\left(a_{2} / \alpha\right)$ is almost $(k, \Gamma)$-internal and as $a_{2}$ is algebraic over $k, \Gamma, a$ then the type $\operatorname{tp}^{t}\left(n_{1} \cdot a_{2} / \alpha\right)$ is also almost $(k, \Gamma)$-internal. As $\alpha$ is algebraic over $k, \Gamma, a_{2}$, the type $\operatorname{tp}^{t}\left(n_{1} / k, \Gamma, a_{2}\right)$ is almost $(k, \Gamma)$-internal. Owing to the independence $n_{1} \underset{k, \Gamma}{t} a_{2}$, we conclude that $\operatorname{tp}^{t}\left(n_{1} / k, \Gamma\right)$ is also almost $(k, \Gamma)$-internal. Then by lemma $(6.12)$ we have that $N_{1}$ is isogenous to a cartesian product of $k$-rational points of an algebraic group defined over $k$ and an interpretable group in $\Gamma$ which is abelian by (5.11).

Acknowledgements. The author thanks his PhD supervisors Amador Martin-Pizarro and Frank Wagner for many motivating discussions. The author would like to thank the anonymous referees for the suggestions, which immensely improved the quality of paper. The author is also grateful to Ayhan Günaydın for very fruitful discussions related to this paper.

\section{REFERENCES}

[1] I.Ben Yaacov, A. Pillay, E. Vassilliev, Lovely pairs of models, Annals of Pure and Applied Logic, 2003.

[2] T. Blossier, A. Martin-Pizarro, De Beaux Groupes, Confl. Math. 6 (2014), 3-13.

[3] T. Blossier, A. Martin-Pizarro, F. Wagner, Géométries Relatives, J. Europ. Math. Soc. 17 (2015), $229-258$.

[4] L. van den Dries and A. Günaydın, The Fields of Real and Complex Numbers with a Small Multiplicative Group, Proc. London Math. Soc. (3) 93 (2006) 43-81.

[5] L. van den Dries and A. Günaydın, Mann Pairs, Trans. Amer. Math. Soc. 362, (2010), 2393-2414.

[6] L. van den Dries and A. Günaydın, Definable sets in Mann Pairs, Comm. Alg. 39, (2011) 2752-2763.

[7] J.H. Evertse, H.P. Schlickewei, W. M. Schmidt, Linear equations in variables which lie in a multiplicative group, Annals of Mathematics, 155 (2002), 807-836. 
[8] M. D. Fried, M. Jarden, Field Arithmetic, A Series of Modern Surveys in Mathematics, Springer-Verlag, 2008.

[9] Haydar Göral, Model Theory of Fields and Heights, Ph.D thesis, Lyon, 2015.

[10] Haydar Göral, Tame Expansions of $\omega$-Stable Theories and Definable Groups, accepted, Notre Dame Journal of Formal Logic.

[11] E. Hrushovski, Contributions to stable model theory, Ph.D thesis, Berkeley, 1986.

[12] E. Hrushovski and A. Pillay, Weakly normal groups, Stud. Logic Found. Math. 122 (1987), 233244.

[13] P. Kowalski and A. Pillay, A Note on Groups Definable in Difference Fields, Proc. of AMS (1) 130 (2002), 205-212.

[14] S. Lang, Algebra, Springer-Verlag, Revised Third Edition, 2005.

[15] H. Mann, On linear relations between roots of unity, Mathematika 12 (1965) 107-117.

[16] D. Marker, Model Theory: An Introduction, Springer-Verlag, New York, 2002.

[17] A. Pillay, Imaginaries in pairs of algebraically closed fields, Annals of Pure and Applied Logic, 2007.

[18] A. Pillay, Geometric Stability Theory, Oxford Logic Guides.

[19] B. Poizat, Groupes Stables.(1987). Traduction anglaise: Stable groups. Mathematical Surveys and Monographs, 87. Amer. Math. Soc. (2001).

[20] B. Poizat, Paires de structures stables, JSL, 48, 239-249, (1983).

[21] K. Tent, M. Ziegler, A Course in Model Theory: Lecture Notes in Logic, ASL, 2012.

[22] F. Wagner, Stable Groups, Lecture Notes of the London Mathematical Society 240, Cambridge University Press.

[23] F. Wagner, Simple Theories, Kluwer Academic Publishers, (2000).

Department of Mathematics, Koç University, Rumelifeneri Yolu, 34450, Sariyer, Istanbul, TURKEY.

E-mail address: hgoral@gmail.com 